\title{
Arrangements of hypersurfaces and Bestvina-Brady groups
}

\author{
Enrique Artal Bartolo, ${ }^{1}$ José Ignacio Cogolludo-Agustín, ${ }^{1}$ \\ and Daniel Matei ${ }^{1,2}$
}

\begin{abstract}
We show that quasi-projective Bestvina-Brady groups are fundamental groups of complements to hyperplane arrangements. Furthermore we relate other normal subgroups of right-angled Artin groups to complements to arrangements of hypersurfaces. We thus obtain examples of hypersurface complements whose fundamental groups satisfy various finiteness properties.
\end{abstract}

Mathematics Subject Classification (2010). 14F35, 20F65, 14F45, 20F36, 55R55, 14N20, $14 \mathrm{~J} 70$.

Keywords. Bestvina-Brady quasi-projective groups, Artin kernels, hyperplane and toric arrangements, quasifibrations.

\section{Introduction}

An important open question in the topology of complex algebraic varieties is J.-P. Serre's problem to characterize fundamental groups of smooth algebraic varieties (cf. [32]): Which finitely presented groups are quasi-projective, i.e. isomorphic to the fundamental group of a smooth connected quasi-projective variety (the complement of a normal crossing divisor in a smooth, connected, complex projective variety)? One could also consider an analogous question for quasi-Kähler groups, that is, for fundamental groups of hypersurface complements in a compact Kähler manifold. On the other hand, one could explore particular versions of Serre's question by restricting either the ambient projective variety or the divisor, or both. For example: Which groups can be realized as fundamental groups

${ }^{1}$ Partially supported by MTM2010-21740-C02-02.

${ }^{2}$ The third author is also partially supported by grant CNCSIS PNII-IDEI 1188/2008 and FMI 53/10 (Gobierno de Aragón). 
of hypersurface (in particular hyperplane) arrangement complements in a projective space? or Which finitely presented groups are projective, i.e. isomorphic to fundamental groups of smooth connected projective varieties?

In this paper we are interested in quasi-projective groups from the point of view of their finiteness properties. It is well known that such groups are finitely presented, but much less is known about their higher dimensional finiteness properties. In [36], C. T. C. Wall introduced general finiteness properties of groups and $\mathrm{CW}$-complexes. A group $G$ is said to be of type $\mathrm{F}_{n}$ (resp. F) if it has an Eilenberg-MacLane complex $K(G, 1)$ with finite $n$-skeleton (resp. finite). Clearly $G$ is finitely generated if and only if it is $\mathrm{F}_{1}$ and finitely presented if and only if it is $\mathrm{F}_{2}$. An interesting example of a finitely generated group which is not finitely presented was given by Stallings in [34]. While Wall's property $\mathrm{F}_{n}$ has a substantial geometric meaning, it is not easily detectable by homological algebraic methods.

In [5], Bieri introduced homological counterparts of Wall's finiteness conditions. A group $G$ is said to be of type $\mathrm{FP}_{n}$ if the trivial $\mathbb{Z} G$-module $\mathbb{Z}$ admits a projective resolution which is finitely generated in dimensions $\leq n$. The condition $\mathrm{FP}_{n}$ is clearly weaker than $\mathrm{F}_{n}$. Note also that $G$ is of type $\mathrm{FP}_{1}$ if and only if it is finitely generated, and that $G$ is of type $\mathrm{FP}_{2}$ if it is finitely presented. But, as shown by Bestvina and Brady [3] $\mathrm{FP}_{2}$ does not imply finite presentation. Nevertheless, if $G$ is $\mathrm{F}_{2}$ then $G$ is $\mathrm{FP}_{n}$ if and only if it is $\mathrm{F}_{n}$.

The first example of a group which is finitely presented but not of type $\mathrm{FP}_{3}$ was given by Stallings in [34]. Afterwards Bieri [4, 5] generalized Stallings' example to an infinite family. In a nutshell, let $\mathbb{F}_{2} \times \cdots \times \mathbb{F}_{2}$ be the direct product of $r+1$ free groups, each of rank 2. Then the kernel $N$ of the map taking each generator to $1 \in \mathbb{Z}$ is of type $\mathrm{FP}_{r}$ but not $\mathrm{FP}_{r+1}$. Stallings considered the cases $r \leq 2$. In [3], Bestvina and Brady generalized the Bieri-Stallings family by presenting a systematic way of constructing groups $N$ of type $\mathrm{F}_{r}$, but not of type $\mathrm{F}_{r+1}$. This construction was further extended in [23, 6], and revisited in [30]. The input is a finite graph $\Gamma$, together with a homomorphism $\chi \rightarrow \mathbb{Z}$ of its associated right-angled Artin group $A_{\Gamma}$. The generalized Bestvina-Brady group $N_{\Gamma}^{\chi}$, or Artin kernel, is then defined as the kernel of the homomorphism $\chi: A_{\Gamma} \rightarrow \mathbb{Z}$, see [30]. The Bestvina-Brady groups from [3] are recovered as $N_{\Gamma}:=N_{\Gamma}^{\chi}$ for the diagonal homomorphism $\chi: A_{\Gamma} \rightarrow \mathbb{Z}$ sending each generator to 1 .

The class of Bestvina-Brady groups $N_{\Gamma}$ which are quasi-projective was determined by Dimca, Papadima and Suciu in [13] as those corresponding to either trees or special multipartite complete graphs $\Gamma$, i.e. $N_{\Gamma}=\mathbb{F}_{n_{0}} \times \ldots \times \mathbb{F}_{n_{r}}$ with either $r \geq 2$ or $n_{i}=1$ for some $i$. In [13], it was proved in particular that the StallingsBieri groups are quasi-projective. It was first noticed by the third named author 
and Suciu in 2004, and pointed out in [28], that the Stallings group may be realized as the fundamental group of the complement of a complex line arrangement in $\mathrm{P}^{2}$. This arrangement (together with a presentation of its fundamental group) was first considered by Arvola in an unpublished work from 1992 (see [31]). An explicit isomorphism between the latter and the Stallings group can be found in [35]. In [21] and [1] we reported on our generalization of this observation. More precisely, we exhibited in [21], for each quasi-projective Bestvina-Brady group $N_{\Gamma}$, a complex line arrangement in $\mathbb{P}^{2}$ having $N_{\Gamma}$ as the fundamental group of the complement, and in [1] higher dimensional hyperplane arrangements realizing just the Stallings-Bieri groups. In this work we will present the geometric construction that was announced in $[21,1]$ (see Theorem 3.2 for more details).

Theorem 1. Let $\Gamma$ be a graph on $v$ vertices, and suppose $N_{\Gamma}$ is a quasi-projective Bestvina-Brady group. Then $N_{\Gamma}$ is the fundamental group of the complement of a hyperplane arrangement $\mathcal{A}_{\Gamma}$ in $\mathbb{P}^{r}$, for some $r<v$ depending only on $\Gamma$.

Theorem 1 answers negatively [2, Question 2.10] from Bestvina's problem list. Indeed, the fundamental group $G=N_{\Gamma}$ of the complement of $\mathcal{A}_{\Gamma}$ cannot have a finite $K(G, 1)$, as long as $H_{r+1}\left(N_{\Gamma}\right)$ has infinite rank. Moreover, as we shall see later in Corollary 3.3, the arrangement $\mathcal{A}_{\Gamma}$ may be defined by real equations. By taking a generic plane section of $\mathbb{P}^{r}$, one can realize $N_{\Gamma}$ as the fundamental group of the complement to a real line arrangement in $\mathbb{P}^{2}$. For another approach to this result we refer to the work of Cohen, Falk and Randell [8].

Our geometric realization of the quasi-projective Bestvina-Brady group $N_{\Gamma}$ as the fundamental group of an arrangement complement $M$, gives more than just the fundamental group of $K=K\left(N_{\Gamma}, 1\right)$. In fact $M$ has the homotopy type of the $r$-skeleton of $K$.

Theorem 2. Let $\mathcal{A}_{\Gamma}$ be a hyperplane arrangement in $\mathrm{P}^{r}$ with complement $M=$ $M_{\Gamma}$ such that $N_{\Gamma}=\pi_{1}(M)$ is a quasi-projective Bestvina-Brady group. Then $M$ has the homotopy type of the $r$-skeleton of $K\left(N_{\Gamma}, 1\right)$. Suppose $N_{\Gamma}$ is not the direct product of free groups. Then

(1) the homology groups $H_{i}(M)$ and $H_{i}\left(N_{\Gamma}\right)$ are isomorphic for $i \leq r$;

(2) the homotopy groups $\pi_{i}(M)$ vanish in the range $1<i<r$;

(3) the homotopy group $\pi_{r}(M)$ does not vanish, in fact its group of coinvariants $\pi_{r}^{*}(M)$ under the $\pi_{1}$-action is isomorphic to $H_{r+1}\left(N_{\Gamma}\right)$. 
One could compare this with the results in [14] where examples are constructed of smooth projective manifolds $M$ of dimension $r \geq 2$ with $\pi_{1}(M)$ having the finiteness properties of the Bestvina-Brady groups, and $\pi_{r}(M)$ the next nonvanishing higher homotopy group.

The results in Theorems 1 and 2 extend to quasi-projective Artin kernels beyond the Bestvina-Brady groups. We will show that an Artin kernel $N_{\Gamma}^{\chi}$ is quasiprojective if $\Gamma$ is a multipartite complete graph with $N_{\Gamma}$ quasi-projective, and the epimorphism $\chi$ of the Artin group $A_{\Gamma}=\mathbb{F}_{n_{0}} \times \cdots \times \mathbb{F}_{n_{r}}$ sends each free factor $\mathbb{F}_{n_{i}}$ to a non-zero integer. More precisely, $N_{\Gamma}^{\chi}$ will be realized as the fundamental group of an arrangement of hypersurfaces in an $r$-dimensional weighted complex projective space whose weights are given by the images under $\chi$ of the generators of $A_{\Gamma}$. In practice though we will work not in the weighted projective space, but in its associated torus. Thus all Artin kernels $N_{\Gamma}^{\chi}$ realized by our construction turn out to be fundamental groups of complements to arrangements of hypertori in an ambient complex algebraic torus. The basic properties of such toric arrangements are similar to those of hyperplane arrangements, see monographs [10] and [26].

The description of the quasi-projective Bestvina-Brady groups provided by Theorems 1 and 2 yields a large class of hyperplane arrangements that exhibit new topological features. More precisely, the affine arrangements in $\mathbb{C}^{r+1}$ induced by the projective arrangements $\mathcal{A}_{\Gamma}$, cannot be hypersolvable since their groups are not of type F. Thus the techniques described in [27] can no longer be used to determine the homotopy and homology groups of these arrangement complements. Compare also with the earlier results of [31], obtained using the Lefschetz hyperplane theorem. This suggests the following open problem.

Problem. Characterize the quasiprojective Artin kernels.

In the following we will explain the motivation and the basic steps for the geometric construction that will produce quasi-projective Artin kernels. Suppose $X$ is a smooth (quasi)-projective variety. Then any finite covering of $X$ is again a (quasi)-projective manifold (see for instance Griffiths and Harris [16, p. 192] for the projective case and Namba [25, Lemma 2.1.2] for the general case using the Grauert-Remmert extension theorem of unramified coverings of complex spaces).

In general, the (quasi)-projectivity property is lost when passing to infinite coverings. Suppose that $\pi_{1}(X)$ admits an epimorphism $v: \pi_{1}(X) \rightarrow \mathbb{Z}$. Let $Y$ be the regular connected infinite cyclic covering of $X$ associated with $v$. We are interested in deciding whether or not $Y$ is a (quasi)-projective manifold. The first obstruction would be the finite presentability of $\pi_{1}(Y)$. We may wonder what other properties of $X$ or $\pi_{1}(X)$ survive after infinite cyclic coverings. For example 
the $K(\pi, 1)$ property for $X$, or other finiteness properties of $\pi_{1}(X)$ such as $\mathrm{F}_{n}, \mathrm{~F}$, or $\mathrm{FP}_{n}$.

In this context, the general problem that motivates our construction is the following one.

Problem. Identify (quasi)-projective manifolds $X$ admitting infinite cyclic coverings which have the homotopy type of a (quasi)-projective manifold. Alternatively, identify quasi-projective groups $G$ having normal subgroups $N$, such that $G / N \cong \mathbb{Z}$, which are again quasi-projective.

A very natural idea is to start with a polynomial mapping $f: \mathbb{C}^{n} \rightarrow \mathbb{C}$, and analyze the restriction $f: X \rightarrow \mathbb{C}^{*}$, where $X=\mathbb{C}^{n} \backslash V(f)$. The bifurcation set $\Lambda=\Lambda_{f}$ of $f$ is the minimal set to be removed so that $f: X \backslash f^{-1}(\Lambda) \rightarrow \mathbb{C}^{*} \backslash \Lambda$ is a locally trivial fibration. Then $\Lambda$ contains $C=C_{f}$ the set of critical values of $f$ (in general only as a proper subset).

Now suppose that $f$ has finitely many singular points, and it behaves like a proper map, in particular the critical set is the bifurcation set of $f$. If $n \geq 3$, then one can obtain, by standard arguments, an exact sequence of groups

$$
1 \longrightarrow \pi_{1}\left(F_{a}\right) \longrightarrow \pi_{1}(X) \longrightarrow \mathbb{Z} \longrightarrow 1,
$$

for any fiber $F_{a}=f^{-1}(a), a \in \mathbb{C}^{*}$ of $f$, whether smooth or singular, see [13, 33].

In the present paper a different approach will be presented. Suppose that both $f$ and $g$ are two polynomial mappings $\mathbb{C}^{n} \rightarrow \mathbb{C}$, and consider the restrictions $f_{\mid T}: T \rightarrow \mathbb{C}^{*}$, where $T=\mathbb{C}^{n} \backslash V(f)$, and $f_{\mid X}: X \rightarrow \mathbb{C}^{*}$, where $X=T \backslash V(g)$.

If $f_{\mid T}: T \rightarrow \mathbb{C}^{*}$ is a trivial fiber bundle, and $V(g)$ is transverse (in the stratified sense) to the fibers $f_{\mid T}^{-1}(a)$ with $a \in \mathbb{C}^{*} \backslash \Lambda$, then $f_{\mid X_{\Lambda}}: X_{\Lambda} \rightarrow \mathbb{C}^{*} \backslash \Lambda, X_{\Lambda}:=$ $X \backslash f^{-1}(\Lambda)$, is again a fiber bundle $\left(\Lambda\right.$ is the bifurcation set of $f: X \rightarrow \mathbb{C}^{*}$ ).

Let $\Sigma$ be the union of the strata in $V(g)$ which are not transverse to $f^{-1}(\Lambda)$, and denote its dimension by $s:=\operatorname{dim} \Sigma$. Then, using Dold-Thom fibration theory one can see that the map $f: X \rightarrow \mathbb{C}^{*}$ behaves like a fibration up to dimension $n-s-1$ (details will be given in Section 5).

Now suppose $X$ is aspherical and $s=0$. It follows that all the fibers $F_{a}=$ $f^{-1}(a)$ share the same homotopy type up to dimension $(n-2)$, in particular, if $n \geq 4$, then they have the same fundamental group. Furthermore, if the monodromy of $f: X \rightarrow \mathbb{C}^{*}$ about the points in $\Lambda$ is trivial, we then have an exact sequence $1 \rightarrow \pi_{1}(F) \rightarrow \pi_{1}(X) \rightarrow \mathbb{Z} \rightarrow 1$. We will show that indeed that is the case for the constructions of interest to us, by analyzing the homotopy short exact sequence $1 \rightarrow \pi_{1}(F) \rightarrow \pi_{1}\left(X_{\Lambda}\right) \rightarrow \pi_{1}\left(\mathbb{C}^{*} \backslash \Lambda\right) \rightarrow 1$. 
The paper is organized as follows: in the first two sections the main objects are introduced. More specifically the group theoretical objects, such as Artin groups, Artin kernels, and their presentations, are described in Section 1. The geometrical objects such as hyperplane and toric arrangements are described in Section 2 together with their homological properties. The main results extending Theorems 1 and 2 are stated in Section 3 and their proofs will be shown in the following two sections. Section 4 describes the arrangements realizing the quasi-projective groups of Section 1. Finally, the homotopical and homological properties of these groups are discussed in Section 5 in terms of arrangements using quasifibration theory.

We would like to thank to the referee for his/her adequate comments and remarks.

\section{Artin kernels}

Let $\Gamma$ be a finite simplicial graph, i.e., $\Gamma:=\left(V_{\Gamma}, E_{\Gamma}\right)$, where $V_{\Gamma}$ is a finite set, say $\{0,1, \ldots, s\}$, and $E_{\Gamma} \subset\left\{A \subset V_{\Gamma} \mid \# A=2\right\}$; let $L_{\Gamma}$ be the flag simplicial complex generated by $\Gamma$, where $J:=\left\{j_{1}, \ldots, j_{k}\right\}$ is a simplex of $L_{\Gamma}$ if and only if the complete graph on the vertices $J$ is a subgraph of $\Gamma$. The right-angled Artin group $A_{\Gamma}$ associated with $\Gamma$ is the group with generators $\sigma_{1}, \ldots, \sigma_{s}$ and relations $\sigma_{i} \sigma_{j}=\sigma_{j} \sigma_{i}$, one for each edge $\{i, j\} \in E_{\Gamma}$ of $\Gamma$.

The Eilenberg-MacLane space $K\left(A_{\Gamma}, 1\right)$ has two remarkable incarnations as toric spaces. First, consider the real $(s+1)$-torus $T=\left(\mathbb{S}^{1}\right)^{s+1}$ with its standard $\mathrm{CW}$-complex structure, where $k$-cells are in bijection with $k$-subsets of $\{0,1, \ldots$, $s\}$. From a simplicial complex $L=L_{\Gamma}$ one constructs the subcomplex $T_{L}$ of $T$, by retaining only the cells corresponding to the simplices of $L$. It is well known that $T_{L}$ is a $K\left(A_{\Gamma}, 1\right)$-space [7]. In the language of [11], $T_{L}$ is the moment-angle complex $Z_{L}\left(\mathbb{S}^{1}\right)$, obtained by gluing subtori of $T$ according to the simplicial structure of $L$. A similar gluing process, but using the complex $(s+1)$-torus $\mathrm{T}^{s+1}=\left(\mathbb{C}^{*}\right)^{s+1}$ and complex subtori accordingly, produces $Z_{L}\left(\mathbb{C}^{*}\right)$, which we denote by $\mathbb{T}_{L}$.

The properties of the construction $Z_{L}$, see [11], ensure that $T_{L}$ and $\mathbb{T}_{L}$ have the same homotopy type. It also follows that $T_{L * M}=T_{L} \times T_{M}$, and respectively $\mathrm{T}_{L * M}=\mathbb{T}_{L} \times \mathbb{T}_{M}$, where $L * M$ is the join of $L$ and $M$.

Example 1.1. Let us consider two standard examples of graphs on $s+1$ vertices, namely, $K_{s+1}$ the complete graph, and $\bar{K}_{s+1}$ the graph with no edges. The flag complexes are respectively an $s$-dimensional simplex and $\bar{K}_{s+1}$ itself. As for the 
associated right-angled Artin groups, $A_{K_{s+1}} \cong \mathbb{Z}^{s+1}$ and $A_{\bar{K}_{s+1}} \cong \mathbb{F}_{s+1}$, the free group of rank $s+1$. The toric complexes are $T_{K_{s+1}}=T \simeq \mathbb{T}=\mathbb{T}_{K_{s+1}}$ and $T_{\bar{K}_{s+1}}=\bigvee^{s+1} \mathbb{S}^{1} \simeq \bigvee^{s+1} \mathbb{C}^{*}=\mathbb{T}_{\bar{K}_{s+1}}$ respectively. Note that $\bigvee^{s+1} \mathbb{C}^{*}$ has the homotopy type of $\mathbb{C}$ punctured $s+1$ times. We denote by $\mathbb{C}_{s+1}^{*}$ an $(s+1)$ punctured $\mathbb{C}$. Thus we have $\mathbb{T}_{\bar{K}_{s+1}} \simeq \mathbb{C}_{s+1}^{*}$.

Example 1.2. Let $\overline{\mathbf{n}}:=\left(n_{0}, \ldots, n_{r}\right)$ be an $(r+1)$-tuple of positive integers, $n_{i} \geq 1$. Denote by $\bar{K}_{\overline{\mathbf{n}}}$ the multipartite graph defined by the join $\bar{K}_{n_{0}} * \cdots * \bar{K}_{n_{r}}$. The associated flag complex $L=L_{\bar{K}_{\overline{\mathbf{n}}}}$ has the homotopy type of a wedge of $m:=$ $\prod_{i=0}^{r}\left(n_{i}-1\right)$ spheres $\bigvee^{m} \mathbb{S}^{r}$. The Artin group $A_{\bar{K}_{\overline{\mathbf{n}}}}$ is the product $\mathbb{F}_{n_{0}} \times \cdots \times \mathbb{F}_{n_{r}}$ of free groups, and $\mathbb{T}_{L} \simeq \mathbb{C}_{n_{0}}^{*} \times \cdots \times \mathbb{C}_{n_{r}}^{*}$.

We will consider here a class of subgroups of the Artin group $A_{\Gamma}$, generalizing the Bestvina-Brady groups of [3]. An Artin kernel is nothing but the kernel of an epimorphism $\chi: A_{\Gamma} \rightarrow \mathbb{Z}$, see [30]. More precisely, given an epimorphism $\chi: A_{\Gamma} \rightarrow \mathbb{Z}$, we denote its kernel by $N_{\Gamma}^{\chi}$. The living flag complex $L_{\Gamma}^{\chi}$ associated to $\chi$ is the flag complex of the full subgraph of $\Gamma$ whose vertices $v$ are those such that $\chi(v) \neq 0$.

Theorem 1.3 ([3, 23, 6, 30]). The Artin kernel $N_{\Gamma}^{\chi}$ is finitely presented if and only if $L_{\Gamma}^{\chi}$ is simply-connected. Moreover, $N_{\Gamma}^{\chi}$ is of type $\mathrm{F}_{r}$ (respectively $\mathrm{FP}_{r}$ ) if and only if the flag complex $L_{\Gamma}^{\chi}$ is $(r-1)$-connected (respectively $(r-1)$-acyclic). In addition, $N_{\Gamma}^{\chi}$ is of type $\mathrm{FP}_{r}$ if and only if $\operatorname{dim}_{\mathbb{K}} H_{\leq r}\left(N_{\Gamma}^{\chi}, \mathbb{K}\right)<\infty$, for any field $\mathbb{K}$.

In this paper we will always assume that all integers $\chi\left(\sigma_{i}\right)$ are non-zero. This implies that $L_{\Gamma}^{\chi}$ in Theorem 1.3 is the usual flag complex $L_{\Gamma}$ on $\Gamma$. Let $\mathbb{T}_{\Gamma}^{\chi} \rightarrow \mathbb{T}_{\Gamma}$ be the infinite cyclic cover determined by $\chi$. Note that $\pi_{1}\left(\mathrm{~T}_{\Gamma}^{\chi}\right) \cong N_{\Gamma}^{\chi}$.

When $\chi$ is the epimorphism $\chi: A_{\Gamma} \rightarrow \mathbb{Z}$ defined by $\chi\left(\sigma_{i}\right):=1$, the kernel $N_{\Gamma}:=\operatorname{ker} \chi$ is known as the Bestvina-Brady group associated with $\Gamma$. As noted above, this group is finitely presented if and only if $L$ is simply-connected. In that case, $N_{\Gamma}$ admits a commutator-relators presentation, as it is shown in [29, Corollary 2.3] (which follows essentially from [12, Corollary 3]). As a consequence, one immediately obtains that $b_{1}\left(N_{\Gamma}\right)=\left|V_{\Gamma}\right|-1$ and $b_{2}\left(N_{\Gamma}\right)=\left|E_{\Gamma}\right|-\left|V_{\Gamma}\right|+1$.

The Artin kernels that interest us most here are those associated with the multipartite graph $\bar{K}_{\overline{\mathbf{n}}}$. For this special case, we introduce now some notation. After a suitable labeling, we denote the set of vertices of $\bar{K}_{\overline{\mathbf{n}}}$ by $\bigcup_{k=0}^{r}\left\{(k, i) \mid 1 \leq i \leq n_{k}\right\}$ where $(k, i)$ is a vertex of $\bar{K}_{n_{k}}$. The corresponding generators of $A_{\bar{K}_{\overline{\mathbf{n}}}}$ are denoted by $\sigma_{k, i}$.

Let $\mathbf{d}:=\left(d_{0}, \ldots, d_{r}\right) \in \mathbb{Z}^{r}$ and consider the epimorphism $\chi_{\mathbf{d}}: A_{\bar{K}_{\overline{\mathbf{n}}}} \rightarrow \mathbb{Z}$ defined by $\chi_{\mathbf{d}}\left(\sigma_{k, i}\right):=d_{k}$. 
Corollary 1.4. The Artin kernel $N_{\overline{\mathbf{n}}}^{\mathbf{d}}:=N_{\bar{K}_{\overline{\mathbf{n}}}}^{\chi_{\mathbf{d}}}$ is of type $\mathrm{F}_{r}$, but not $\mathrm{FP}_{r+1}$, if all $n_{i}>1$.

Proof. Since $L_{\bar{K}_{\overline{\mathbf{n}}}} \simeq \bigvee^{m} \mathbb{S}^{r}$ is $(r-1)$-connected, but $H_{r}\left(L_{\bar{K}_{\overline{\mathbf{n}}}}\right) \neq 0$, it follows from Theorem 1.3 that $N_{\overline{\mathbf{n}}}^{\mathbf{d}}$ is of type $\mathrm{F}_{r}$, but not $\mathrm{FP}_{r+1}$, if all $n_{i}>1$.

Note that, as soon as $r \geq 2$, the Artin kernel $N_{\overline{\mathbf{n}}}^{\mathbf{d}}$ is finitely presented and thus the $\mathrm{F}_{m}$ and $\mathrm{FP}_{m}$ properties coincide in this case. For $N=N_{\bar{K}_{\overline{\mathbf{n}}}}$ one obtains

$$
b_{1}(N)=\sum_{i=0}^{r} n_{i}-1, \quad b_{2}(N)=\sum_{0 \leq i<j \leq r} n_{i} n_{j}-\sum_{i=0}^{r} n_{i}+1 .
$$

Given a group $G$ of type $\mathrm{FP}_{m}$, for some integer $m$, we recall the definition of the Poincaré series of $G$, as the generating function of its Betti numbers, that is, $P_{G}(t):=\sum_{k=0}^{\infty} b_{k}(G) t^{k} .>$ From the results of [30] it follows that the truncated Poincaré polynomials of $A_{\Gamma}$ and $N_{\Gamma}$, where $\Gamma$ is a multipartite graph, are related as

$$
P_{A_{\Gamma}}(t) \equiv(1+t) P_{N_{\Gamma}}(t) \bmod t^{r+1} .
$$

It is easily seen that the $r$-th truncation of the Poincaré polynomial of $A=A_{\bar{K}_{\overline{\mathbf{n}}}}$ is given by

$$
P_{A}(t) \equiv \prod_{i=0}^{r}\left(1+n_{i} t\right) \quad \bmod t^{r+1} .
$$

This gives the Betti numbers $b_{k}$ of $N=N_{\bar{K}_{\overline{\mathbf{n}}}}$, in the range $0 \leq k<r$ :

$$
b_{k}(N)=\sum_{p=0}^{k}(-1)^{k-p} \sum_{0 \leq i_{1}<\cdots<i_{p} \leq r} n_{i_{1}} \ldots n_{i_{p}} .
$$

1.5. Finite presentations of $N^{\chi}$. A finite presentation for $N_{\Gamma}$ can be obtained using the Reidemeister-Schreier method; a presentation of $N_{\Gamma}$ for arbitrary $\Gamma$ can be found in [12]. Let us compute presentations for $N^{\chi}=N_{\overline{\mathbf{n}}}^{\mathbf{d}}$, where $r \geq 2$, $n_{0}, n_{1}, \ldots, n_{r} \geq 2$ and $\operatorname{gcd}\left(d_{0}, d_{1}, \ldots, d_{r}\right)=1$. Fix $a_{k} \in \mathbb{Z}$ so that $\sum_{k=0}^{r} a_{k} d_{k}=$ 1 and define $\tau:=\prod_{k=0}^{r} \sigma_{k, 1}^{a_{k}}$; note that $\chi(\tau)=1$. Since $\sigma_{k, 1}, \sigma_{\ell, 1}$ commute whenever $k \neq \ell$, note that $\tau^{m}=\prod_{k=0}^{r} \sigma_{k, 1}^{m a_{k}}, m \in \mathbb{Z}$. We can add $\tau$ to the generators of $A_{\bar{K}_{\overline{\mathbf{n}}}}$, adding the following relations (some of them are redundant but useful):

$$
\tau=\prod_{k=0}^{r} \sigma_{k, 1}^{a_{k}}, \quad\left[\tau, \sigma_{k, 1}\right]=1, \quad k=0, \ldots, r .
$$


Applying the Reidemeister-Schreier method, a set of generators of $N^{\chi}$ is given by

$$
\alpha_{k, i ; m}:=\tau^{m} \sigma_{k, i} \tau^{-m-d_{k}}, \quad 0 \leq k \leq r, 1 \leq i \leq n_{k}, m \in \mathbb{Z} .
$$

The commutativity relations in (1.1) imply that $\alpha_{k, 1 ; m}$ does not depend on $m$ and hence we denote them by $\beta_{k}$. The remaining relation in (1.1) gives the following one for $N^{\chi}$ :

$$
\prod_{k=0}^{r} \beta_{k}^{a_{k}}=1 .
$$

The relations $\left[\sigma_{k, i}, \sigma_{\ell, j}\right]=1, k \neq \ell$ give the following ones in the kernel $N^{\chi}$

$$
\alpha_{k, i ; m} \alpha_{\ell, j ; m+d_{k}}=\alpha_{\ell, j ; m} \alpha_{k, i ; m+d_{\ell}}, \quad \text { if } k \neq \ell .
$$

If $i=j=1$, (1.3) simplifies as

$$
\left[\beta_{k}, \beta_{\ell}\right]=1, k \neq \ell
$$

If $i=1<j,(1.3)$ simplifies as

$$
\alpha_{\ell, j ; m+d_{k}}=\beta_{k}^{-1} \alpha_{\ell, j ; m} \beta_{k},
$$

for all $m \in \mathbb{Z}, k \in\{0, \ldots, r\}, \ell \neq k, j \in\left\{2, \ldots, n_{\ell}\right\}$. Let us set

$$
e_{\ell}:=\operatorname{gcd}\left\{d_{k} \mid k \neq \ell\right\}
$$

and choose integers $a_{k, \ell}, a_{k, k}:=0$, such that

$$
e_{\ell}=\sum_{k=0}^{r} a_{k, \ell} d_{k}
$$

Clearly, using relations (1.5), we can reduce the infinite set of generators

$$
\left\{\alpha_{k, i ; m} \mid m \in \mathbb{Z}, k \in\{0, \ldots, r\}, 1 \leq i \leq n_{k}\right\}
$$

to the finite subset

$$
\left\{\beta_{k} \mid 1 \leq k \leq r\right\} \cup\left\{\alpha_{k, j ; p} \mid 0 \leq p<e_{k}, 0 \leq k \leq r, 1<j \leq n_{k}\right\} .
$$

Let us denote $\gamma_{\ell}:=\prod_{k=0}^{r} \beta_{k}^{a_{k, \ell}}$. Then, we obtain

$$
\alpha_{\ell, j ; m+e_{\ell}}=\gamma_{\ell}^{-1} \alpha_{\ell, j ; m} \gamma_{\ell}
$$

for all $m \in \mathbb{Z}, \ell \in\{1, \ldots, r\}, j \in\left\{2, \ldots, n_{\ell}\right\}$. Denote

$$
f_{k, \ell}:=\frac{d_{k}}{e_{\ell}}, \quad k \neq \ell
$$


Combining (1.5)-(1.7), we obtain

$$
\left[\alpha_{\ell, j ; m}, \gamma_{\ell}^{f_{k, \ell}} \beta_{k}^{-1}\right]=1,
$$

with $k \in\{0, \ldots, r\}, \ell \neq k, j \in\left\{2, \ldots, n_{\ell}\right\}, m \in\left\{0, \ldots, e_{k}-1\right\}$. Consider now the relations (1.3) for $i, j>1$,

$$
\alpha_{k, i ; m} \beta_{k}^{-1} \alpha_{\ell, j ; m} \beta_{k}=\alpha_{\ell, j ; m} \beta_{\ell}^{-1} \alpha_{k, i ; m} \beta_{\ell}, \quad \text { if } k \neq \ell .
$$

Given $k \neq \ell$, fix $m \neq k, \ell$ such that $d_{m}$ is minimal; this is possible since $r \geq 2$. Then it is enough to consider relations (1.9) for $m \in\left\{0, \ldots, d_{m}-1\right\}$.

The presentation of $N^{\chi}$ admits (1.6) as system of generators and (1.2), (1.4), (1.8), and (1.9) as a system of relators.

It seems plausible that the approach of Dicks and Leary from [12], further simplified in [29], can be used to produce finite presentations for the Artin kernels $N_{\Gamma}^{\chi}$ that would involve the combinatorics of the flag complex of $L_{\Gamma}$ in a precise geometric way, but we have not been able to determine such presentations.

Example 1.6. If $d_{0}=1$, we can choose $\tau=\sigma_{0,1}$, i.e. $a_{0}=1$ and $a_{k}=0$ if $k>0$. We get $\beta_{0}=1, e_{k}=1$ if $k>0$, and hence $\gamma_{k}=1$ if $k>0$. Then $N^{\chi}$ is generated by

$$
\alpha_{k, i ; m}=\sigma_{0,1}^{m} \sigma_{k, i} \sigma_{0,1}^{-m-d_{k}},
$$

for

$$
\left\{(0, i ; m) \mid 1<i \leq n_{0}, 0 \leq m<e_{0}\right\} \cup\left\{(k, i ; 0) \mid 0<k \leq r, 1 \leq i \leq n_{k}\right\} .
$$

Let us denote $\mu_{i, m}:=\alpha_{0, i ; m}$ and $v_{k, i}:=\alpha_{k, i ; 0}(k>0)$. Note also that if $k \neq \ell$ and both are different from 0 then, we can choose $m=1$. The relations are

$$
\begin{array}{rlrl}
{\left[v_{k, 1}, v_{\ell, 1}\right]} & =1, & & 0<k<\ell, \\
{\left[\mu_{i, m}, \gamma_{0}^{\frac{d_{k}}{e_{0}}} v_{k, 1}^{-1}\right]} & =1, & & 1<i \leq n_{0}, 0 \leq m<e_{0}, 0<k \leq r, \\
{\left[v_{k, i}, v_{\ell, j}\right]} & =1, & & 0<k, \ell \leq r, k \neq \ell, 1 \leq i \leq n_{k}, 1 \leq j \leq n_{\ell}, \\
{\left[\mu_{j, m}, v_{k, i} v_{k, 1}^{-1}\right]} & =1 & & 0<k \leq r, 1 \leq i \leq n_{k}, \\
& 1<j \leq n_{0}, 0 \leq m<d_{0},
\end{array}
$$

where

$$
\mu_{j, m+e_{1}}:=\gamma_{0}^{-1} \mu_{j, m} \gamma_{0}
$$




\section{Hypersurface arrangements and their complements}

In this section we introduce the arrangements of hypersurfaces that will be needed in the sequel. We will consider two classes: hyperplane arrangements and toric arrangements. In each case, we will explain how the homology of complements to such arrangements may be computed in terms of their combinatorics. We will end with computations of the Poincaré polynomials for the arrangements that we will later relate with Artin kernels.

2.1. Hyperplane arrangements. An arrangement of hyperplanes is a collection $\mathcal{A}$ of hyperplanes in a projective, or affine space. An arrangement $\mathcal{A}$ in $\mathbb{P}^{r}$ may be viewed as a central arrangement in $\mathbb{C}^{r+1}$. If $M$ is the complement of $\mathcal{A}$ in $\mathbb{P}^{r}$, and $M^{\prime}$ the complement in $\mathbb{C}^{r+1}$, we have that $M^{\prime}$ is homeomorphic to $M \times \mathbb{C}^{*}$. It follows that

$$
P\left(M^{\prime}, t\right)=(1+t) P(M, t),
$$

and $\pi_{1}\left(M^{\prime}\right)=\pi_{1}(M) \times \mathbb{Z}$.

The Poincaré polynomial of a complement to a hyperplane arrangement $\mathcal{A}$ is completely determined by its intersection poset $L(\mathcal{A})$, which consists of all nonempty intersections among hyperplanes in $\mathcal{A}$ ordered by reversed inclusion and ranked by codimension.

More precisely, if $\mathcal{A}$ is an affine hyperplane arrangement, the characteristic polynomial of the intersection poset $L(\mathcal{A})$ is defined by

$$
\chi(\mathcal{A}, q):=\sum_{S \in L(\mathcal{A})} \mu(S) q^{\operatorname{dim} S},
$$

where $\mu: L(\mathcal{A}) \rightarrow \mathbb{Z}$ is the Möbius function of the ranked poset $L(\mathcal{A})$, see [26], which satisfies

$$
P(M, t)=t^{r+1} \chi\left(\mathcal{A},-t^{-1}\right) .
$$

The best understood arrangements are those of supersolvable type, see [26]. The complement $M$ of such an arrangement seats at the top of a tower of linear fiber bundles with fiber a punctured complex plane. In particular $M$ is a $K(G, 1)$ space for $G:=\pi_{1}(M)$. A more general class, comprising both supersolvable and generic arrangements, is that of hypersolvable arrangements, see [20]. The group $G$ of a hypersolvable arrangement $\mathcal{A}$ is still of type $\mathrm{F}$, as it is also the group of a supersolvable arrangement $\hat{\mathcal{A}}$ to which $\mathcal{A}$ deforms. 
A class of arrangements that contains both hypersolvable and non-hypersolvable examples is that of graphic arrangements. We refer to [27, 28] for details and further discussion. One associates to a graph $\mathcal{G}=(\mathcal{V}, \mathcal{E})$ on a set of vertices $\mathcal{V}:=\{0, \ldots, r\}$, the arrangement $\mathcal{A}_{\mathcal{G}}$ in $\mathbb{C}^{r+1}$ of hyperplanes $H_{i, j}=\left\{w_{i}=w_{j}\right\}$ indexed by all edges $\{i, j\}$ of $\mathcal{G}$. Any $\mathcal{A}_{\mathcal{G}}$ is a sub-arrangement of the braid arrangement, which corresponds to the complete graph $K_{r+1}$. Denote by $c_{p}$ the number of complete subgraphs $K_{p+1}$ of $\mathcal{G}$.

A graphic arrangement $\mathcal{A}_{\mathcal{G}}$ is supersolvable if and only if $\mathcal{G}$ is a chordal graph (i.e. every circuit of length $\geq 4$ in $\mathcal{G}$ has a chord). For example, an arrangement $\mathcal{A}_{\mathcal{G}}$ with $c_{2}=0$ is hypersolvable. Suppose $\mathcal{G}$ contains no complete graph $K_{4}$ as a subgraph, that is $c_{3}=0$. A sufficient condition for such an arrangement $\mathcal{A}_{\mathcal{G}}$ to be non-hypersolvable is that $0<c_{1} \leq 2 c_{2}$.

Example 2.1. Let $\mathcal{G}=W_{r}$ be the (wheel) graph on $r+1$ vertices obtained by coning an $r$-cycle. In this case, $\mathcal{A}_{W_{r}}$ is defined, after the linear change of coordinates $z_{0}=w_{0}, z_{i}=w_{i}-w_{0}, 1 \leq i \leq r$ by $z_{1} \ldots z_{r}\left(z_{1}-z_{2}\right) \ldots\left(z_{r-1}-z_{r}\right)\left(z_{r}-z_{1}\right)$. Then $c_{1}=2 r, c_{2}=r$, and $c_{3}=0$, if $r>3$. Thus $c_{1}=2 c_{2}$, and so $\mathcal{A}_{W_{r}}$ is non-hypersolvable and decomposable, see [28] for a definition. We shall see that the group of the arrangement $\mathcal{A}_{W_{r}}$ is not of type $\mathrm{F}_{r}$.

2.2. Toric arrangements. Let $v$ be a character of the complex algebraic torus $\mathbb{T}^{r+1}=\left(\mathbb{C}^{*}\right)^{r+1}$ and $a \in \mathbb{C}^{*}$. The pair $(v, a)$ defines a hypersurface $H_{v, a}=$ $\left\{\mathbf{x} \in \mathbb{T}^{r+1} \mid v(\mathbf{x})=a\right\}$. Note that if $v(\mathbf{x})=x_{0}^{d_{0}} \cdot \ldots \cdot x_{r}^{d_{r}}$, then $H_{v, a}$ has $d:=\operatorname{gcd}\left(d_{0}, \ldots, d_{r}\right)$ connected components, which are hypertori.

A finite set $\mathcal{A}$ of such hypertori in $\mathrm{T}^{r+1}$ is called a toric arrangement. Much of the combinatorial and topological theory of such arrangements parallels that of hyperplane arrangements, see [10]. The de Rham cohomology ring of the complement was determined in [9].

Furthermore, the Poincaré polynomial of the complement $M=\mathrm{T}^{r+1} \backslash V(\mathcal{A})$ and the characteristic polynomial of the intersection poset $L(\mathcal{A})$ of $\mathcal{A}$, are related by

$$
P(M, t)=(-t)^{r+1} \chi\left(\mathcal{A},-t^{-1}-1\right),
$$

as shown in [24, Corollary 5.12].

Remark 2.2. Let $\mathcal{H}$ be a hyperplane arrangement in $\mathbb{C}^{r+1}$ defined by equations of the form $z_{i}-a z_{j}=0$, with $a \in \mathbb{C}^{*}$. Consider the hyperplane arrangement $\mathcal{A}$ obtained by adding the coordinate hyperplanes to $\mathcal{H}$. If $\mathcal{T}$ is the toric arrangement in $\mathrm{T}^{r+1}$ obtained by the restriction of $\mathcal{H}$, then the complement of $\mathcal{T}$ in $\mathrm{T}^{r+1}$ may 
be seen as the complement of $\mathcal{A}$ in $\mathbb{C}^{r+1}$. The equality of Poincaré polynomials gives the following relation between characteristic polynomials

$$
\chi(\mathcal{A}, q)=(-1)^{r+1} \chi(\mathcal{T}, q-1)
$$

2.3. General position toric arrangements. We focus now on a particular class of toric arrangements, which consists of general position toric arrangements and some mild deformations of them. Our goal is to compute the Poincaré polynomials of such arrangements.

We start by analyzing an example illustrating all the features of the general case.

Example 2.3. Let $\mathcal{A}_{a}=\left\{H_{0, a}, H_{1}, \ldots, H_{r}\right\}$ be the toric arrangement in $\mathbb{T}^{r}$ defined by the hypertori $H_{0}=H_{0, a}=\left\{x_{1} \cdot \ldots \cdot x_{r}=a\right\}$ and $H_{i}=\left\{x_{i}=1\right\}$, $1 \leq i \leq r$, for some $a \in \mathbb{C}^{*}$.

Note that the hypertori in $\mathcal{A}_{a}$ intersect in general position, except maybe if we consider the intersection of all $r+1$ of them. Indeed, if $H_{I}$ denotes the intersection $\bigcap_{i \in I} H_{i}$, and $I$ is a proper subset of $\{0, \ldots, r\}$, then $H_{I}$ is a connected torus of codimension $|I|$. Now $\bigcap_{i=0}^{r} H_{i}$ is either empty if $a \neq 1$, or the point $(1, \ldots, 1) \in \mathbb{T}$. It follows that the intersection poset $L\left(\mathcal{A}_{a}\right)$ is either the truncation of the lattice of subsets of $\{0, \ldots, r+1\}$ (maximal element removed), if $a \neq 1$, or the obvious collapsing of this truncation for $a=1$. The calculation of the characteristic polynomial of $\mathcal{A}_{a}$ is then immediate:

$$
\chi\left(\mathcal{A}_{a}, q\right)= \begin{cases}\sum_{k=0}^{r}(-1)^{k}\left(\begin{array}{c}
r+1 \\
k
\end{array}\right) q^{r-k} & \text { if } a \neq 1, \\
\sum_{k=0}^{r}(-1)^{k}\left(\begin{array}{c}
r+1 \\
k
\end{array}\right) q^{r-k}-(-1)^{r+1} & \text { if } a=1 .\end{cases}
$$

After evaluating at $q=-\frac{1+t}{t}$, we obtain the Poincaré polynomials

$$
P\left(M\left(\mathcal{A}_{a}\right), t\right)= \begin{cases}\frac{(2 t+1)^{r+1}-t^{r+1}}{t+1} & \text { if } a \neq 1, \\ (2 t+1) \frac{(2 t+1)^{r}-t^{r}}{t+1} & \text { if } a=1 .\end{cases}
$$

Note that the complements $M\left(\mathcal{A}_{a \neq 1}\right)$ differ from $M\left(\mathcal{A}_{1}\right)$ only in their top Betti number $b_{r}$, as $P\left(M\left(\mathcal{A}_{a}\right), t\right)-P\left(M\left(\mathcal{A}_{1}\right), t\right)=t^{r}$. 
We now treat the general case. We work in $\mathbb{T}^{r+1}$ and fix a character $v$ such that $v(\mathbf{x})=x_{0}^{d_{0}} \cdot \ldots \cdot x_{r}^{d_{r}}$ with $\operatorname{gcd}\left(d_{0}, \ldots, d_{r}\right)=1$. Consider the connected $r$-torus $\mathbb{T}_{v, a}=\{v(x)=a\}$ in $\mathbb{T}^{r+1}$ for $a \in \mathbb{C}^{*}$. Let $\mathbf{n}:=\left(n_{0}, n_{1}, \ldots, n_{r}\right)$ and $m_{i}:=n_{i}-1$. We want to compute the Poincaré polynomial of the complement in $\mathbb{T}_{v, a}$ of the arrangement $\mathcal{A}_{v, a}^{\mathbf{n}}(\boldsymbol{\alpha})$ consisting of the hypertori $H_{i}^{j}=\left\{x_{i}=\alpha_{i, j}\right\}$, $0 \leq i \leq r, 1 \leq j \leq m_{i}$. As we shall see, $\mathcal{A}_{v, a}^{\mathbf{n}}(\boldsymbol{\alpha})$ depends on the data $\boldsymbol{\alpha}:=\left(\alpha_{i, j}\right)$. Denote by $\Lambda:=\left\{v\left(\alpha_{0, j_{0}}, \ldots, \alpha_{r, j_{r}}\right) \mid 1 \leq j_{i} \leq m_{i}, 0 \leq i \leq r\right\}$. We will also use the following notation: $[r]=\{0, \ldots, r\}$, and for any $I \subseteq[r], d_{I}:=\operatorname{gcd}\left\{d_{i}, i \in I\right\}$, $m_{I}:=\prod_{i \in I} m_{i}$ and $\bar{I}:=[r] \backslash I$.

Proposition 2.4. Let $M_{a}:=M\left(\mathcal{A}_{v, a}^{\mathbf{n}}(\boldsymbol{\alpha})\right)$ be the complement of $\mathcal{A}_{v, a}^{\mathbf{n}}(\boldsymbol{\alpha})$ in $\mathbb{T}_{v, a}$. Suppose $a \notin \Lambda$. Then the Poincaré polynomial of the complement is equal to

$$
P\left(M_{a}, t\right)=\sum_{k=0}^{r} t^{k}(1+t)^{r-k} \sum_{I \subseteq[r],|I|=k} d_{\bar{I}} m_{I} .
$$

In particular, the $i$-th Betti number of $M_{a}$ is

$$
b_{i}\left(M_{a}\right)=\sum_{l=r-i}^{r}\left(\begin{array}{c}
l \\
r-i
\end{array}\right) \sum_{|I|=r-l} d_{\bar{I}} m_{I} .
$$

Furthermore, if $\lambda \in \Lambda$ then $P\left(M_{\lambda}, t\right) \neq P\left(M_{a}, t\right)$. More precisely, we have

$$
b_{i}\left(M_{a}\right)=b_{i}\left(M_{\lambda}\right) \text { fol all } i<r \text {, and } b_{r}\left(M_{a}\right)>b_{r}\left(M_{\lambda}\right) .
$$

Proof. We compute the characteristic polynomial $\chi(\mathcal{A}, q)$ of the toric arrangement $\mathcal{A}=\mathcal{A}_{v, a}^{\mathbf{n}}(\alpha)$. We claim that the hypertori $H_{i}^{j}$ intersect in general position, except maybe if we consider intersections of $r+1$ of them.

First note that $H_{i}^{j^{\prime}}$ and $H_{i}^{j^{\prime \prime}}$ never intersect if $j^{\prime} \neq j^{\prime \prime}$. Then denote by $H_{I}^{\mathbf{j}}$ the intersection

$$
H_{I}^{\mathbf{j}}=\mathrm{T}_{v, a} \cap \bigcap_{i \in I} H_{i}^{j_{i}}, \quad \text { for } I \subseteq[r], \mathbf{j}=\left(j_{i}\right)_{i \in I} \in \prod_{i \in I}\left\{1, \ldots, m_{i}\right\} .
$$

If $|I| \leq r$, then it can be easily seen that $H_{I}^{\mathbf{j}}$ is non-empty and of codimension $r-|I|$, and it consists of $d_{\bar{I}}$ connected components. If $a \notin \Lambda$, then $H_{[r]}^{\mathbf{j}}$ is empty for all $\mathbf{j}$, and so it does not appear in $L(\mathcal{A})$. Moreover, the Möbius function is readily computed as $\mu\left(H_{I}^{\mathbf{j}}\right)=(-1)^{|I|}$.

Summing up the Möbius function contributions for all the connected components of $H_{I}^{\mathbf{j}}$ one obtains

$$
\chi(\mathcal{A}, q)=\sum_{k=0}^{r}(-1)^{k} q^{r-k} \sum_{I \subseteq[r],|I|=k} d_{\bar{I}} m_{I},
$$


which, after the substitution $q=-t^{-1}-1$, gives the Poincaré polynomial of the $M_{a}$. The formula for the Betti numbers follows after a routine rearrangement of the sum.

Consider now the case $\lambda \in \Lambda$. It is readily seen that the positive dimensional intersections $H_{I}^{\mathbf{j}}$ have the same description as in the generic case. Thus the coefficient of $q^{r-k}$ in $\chi(\mathcal{A}, q)$ will stay the same if $k<r$, hence the equality in (2.1) follows.

The only difference may appear in the independent term, which comes from codimension $r$, i.e. points. To that coefficient only the cardinality of $H_{I}^{\mathbf{j}}$ with $|I|$ equals $r$ or $r+1$ may contribute. By hypothesis $H=H_{[r]}^{\mathbf{j}} \neq \emptyset$ for some $\mathbf{j}$, whereas for $a \notin \Lambda, H_{[r]}^{\mathbf{j}}=\emptyset$, for all $\mathbf{j}$. Such $H$ consists in one point and $\mu(H)=r(-1)^{r}$. Also note that $H$ results from the collapsing of $r+1$ points (each one in $H_{\bar{\iota}}^{\mathbf{j}}{ }_{i}$, where $\mathbf{j}_{i}$ is obtained by forgetting $j_{i}$ in $\mathbf{j}$ ). If these $r+1$ points were different, they would contribute with $\mu=(-1)^{r}(r+1)$, which proves the last assertion.

\section{Main Theorems}

In this section we collect the main results of the paper. The proofs will be given in the last two sections. The quasi-projective Bestvina-Brady groups were determined in [13].

Theorem 3.1 ([13]). Let $\Gamma$ be a finite graph. Suppose the Bestvina-Brady group $N_{\Gamma}$ associated to $\Gamma$ is quasi-projective. Then $\Gamma$ is either a tree, or a complete multipartite graph $K_{n_{0}, \ldots, n_{r}}$, with either some $n_{i}=1$ or all $n_{i} \geq 2$ and $r \geq 2$.

We shall make this statement more precise, by showing that all quasi-projective groups $N_{\Gamma}$ are in fact fundamental groups of hyperplane arrangement complements in $\mathrm{P}^{r}$.

Theorem 3.2. Any quasi-projective Bestvina-Brady group $N_{\Gamma}$ is a hyperplane arrangement group for an arrangement in $\mathrm{P}^{r}$.

In the first case, $N_{\Gamma}$ is simply a free group of rank $v-1$ ( $v$ is the number of vertices of the tree), which is known to be the fundamental group of the complement in $\mathrm{P}^{1}$ of $v$ points.

The second case will interest us most. Let $\Gamma=K_{\mathbf{n}}$, with $\mathbf{n}:=\left(n_{0}, \ldots, n_{r}\right)$. The first part is again easy. Assume $n_{i}=1$ for $0 \leq i \leq s$ and $n_{i}>1$ for $s<i \leq r$. Then it is readily seen that $N_{K_{\mathbf{n}}} \cong \mathbb{Z}^{s} \times \mathbb{F}_{n_{s+1}} \times \cdots \times \mathbb{F}_{n_{r}}$. This group 
is clearly the fundamental group of the complement of a hyperplane arrangement in $\mathbb{C}^{r}=\mathbb{P}^{r} \backslash\left\{z_{0}=0\right\}$ with equation

$$
z_{i}=\alpha_{i}, \quad 1 \leq i \leq s, \quad z_{i}=\alpha_{i, j}, \quad s+1 \leq i \leq r, \quad 1 \leq j \leq n_{i},
$$

for generic choices of $\alpha_{i}, \alpha_{i, j}$. Taking a generic plane section it can be seen as the fundamental group of the complement of a line arrangement. Consider $r-s$ distinct directions in $\mathbb{C}^{2}$ and take $n_{i}$ lines parallel to the $s+i^{\text {th }}$ direction, and $s$ other lines in general position.

So only the case $N_{K_{\mathrm{n}}}$, with all $n_{i} \geq 2$ and $r \geq 2$, is left. We will show in the next section that $N_{K_{\mathbf{n}}}$ is the fundamental group of the complement in $\mathbb{P}^{r}$ to the hyperplane arrangement $\mathcal{A}_{a}^{\mathbf{n}}$ defined by the polynomial

$$
z_{0} \cdot \ldots \cdot z_{r} \cdot \prod_{j=1}^{n_{0}-1}\left(a z_{0}-\alpha_{0, j} z_{1}\right) \cdot\left(\prod_{i=1}^{r-1} \prod_{j=1}^{n_{i}-1}\left(z_{i}-\alpha_{i, j} z_{i+1}\right)\right) \cdot \prod_{j=1}^{n_{r}-1}\left(z_{r}-\alpha_{r, j} z_{0}\right)
$$

A generic plane section provides a line arrangement with a precise combinatorics.

Corollary 3.3. The group $N_{K_{\mathbf{n}}}$ can be realized as $\pi_{1}\left(\mathbb{P}^{2} \backslash \mathcal{A}_{\mathbf{n}}\right)$, where $\mathcal{A}_{\mathbf{n}}$ is a line arrangement in $\mathbb{P}^{2}$ consisting of $\left(n_{0}+1\right) \cdot \ldots \cdot\left(n_{r}+1\right)$ lines, formed by $r$ lines in general position cutting out a polygon with $r+1$ sides which have at vertex $i$ another $n_{i}-1$ lines that intersect transversally among themselves.

If $\Gamma=K_{n_{0}, \ldots, n_{r}}$ then the right-angled Artin group $A_{\Gamma}$ is the product of free groups $\mathbb{F}_{n_{0}} \times \cdots \times \mathbb{F}_{n_{r}}$. Using the same arguments, one can extend this result to other subgroups of $A_{\Gamma}$, namely to the generalized Bestvina-Brady group $N_{\mathbf{n}}^{\mathbf{d}}$, where $\mathbf{n}=\left(n_{0}, \ldots, n_{r}\right)$, and $\mathbf{d}=\left(d_{0}, \ldots, d_{r}\right)$ with $\operatorname{gcd}\left(d_{i}\right)=1$.

Theorem 3.4. Any generalized Bestvina-Brady group $N_{\mathbf{n}}^{\mathbf{d}}$ can be realized as the fundamental group of the complement to an algebraic hypersurface in the weighted projective space $\mathbb{P}^{r}(\mathbf{d})$.

In fact, it is more convenient to view this complement space as obtained from an algebraic $r$-torus by removing $\sum_{i=0}^{r}\left(n_{i}-1\right)$ hypertori. More precisely, we will consider the toric arrangement $\mathcal{A}_{\mathbf{n}, a}^{\mathbf{d}}(\boldsymbol{\alpha})$ from Section 2. In certain cases, as we shall see later, it is still possible to realize this toric arrangement complement as a hypersurface complement in $\mathbb{P}^{r}$, but in general this hypersurface is not the union of hyperplanes. Now recall that a $K(\pi, 1)$ space for $N_{\mathbf{n}}^{\mathbf{d}}$ may be chosen to be the infinite cyclic cover $\mathbb{T}_{K_{n}}^{\chi^{d}}$ of the product $\mathbb{T}_{K_{n}}$ of tori. Then, by putting together the geometric realizability of $N_{\mathbf{n}}^{\mathbf{d}}$ from Section 4 and its higher dimensional consequences from Section 5, we obtain the following. 
Theorem 3.5. Let $M=M\left(\mathcal{A}_{\mathbf{n}, a}^{\mathbf{d}}(\alpha)\right)$ be the complement of the toric arrangement defined above, where $r \geq 2, n_{i}>1$, and $a \notin \Lambda$. Then we have the following:

(1) the r-skeleton of $\mathrm{T}_{K_{\mathbf{n}}}^{\chi^{\mathrm{d}}}$ has the homotopy type of $M$;

(2) the Artin kernel $N_{\mathbf{n}}^{\mathbf{d}}$ is isomorphic to $\pi_{1}(M)$;

(3) the homotopy groups $\pi_{i}(M)$ vanish in the range $1<i<r$;

(4) the homology groups $H_{i}(M)$ and $H_{i}\left(N_{\mathbf{n}}^{\mathbf{d}}\right)$ are isomorphic for $i \leq r$.

\section{A geometric construction}

Suppose $T^{r+1} \backslash D$ is a hypersurface complement in a complex torus of dimension $r+1$. If $f: \mathbb{T}^{r+1} \backslash D \rightarrow \mathbb{C}^{*}$ is a polynomial function then let $\Lambda_{f} \subset \mathbb{C}^{*}$ be the bifurcation set of $f$, that is the smallest set $\Lambda$ such that $f$ is a locally trivial fibration over the complement of $\Lambda$.

In this section we will determine the bifurcation set $\Lambda_{f}$ for certain monomial maps defined on $\mathrm{T}^{r+1} \backslash D$ for which $D$ is a union of hypertori of $\mathrm{T}^{r+1}$, that is a toric arrangement complement.

More precisely, given $(r+1)$-tuples $\mathbf{n}:=\left(n_{i}\right)_{i=0}^{r}$ and $\mathbf{d}:=\left(d_{i}\right)_{i=0}^{r}$ of positive integers, with $\operatorname{gcd} \mathbf{d}=1$, let $p:=p^{\mathbf{d}}$ denote the multiplication map,

$$
p: \mathbb{T}^{r+1} \longrightarrow \mathbb{C}^{*}, \quad p\left(x_{0}, \ldots, x_{r}\right):=x_{0}^{d_{0}} \cdot \ldots \cdot x_{r}^{d_{r}} .
$$

Now, let

$$
f:=f_{\mathbf{n}}^{\mathbf{d}}: X \longrightarrow \mathbb{C}^{*}
$$

denote the restriction of $p$ to the complement $X:=\mathbb{T}^{r+1} \backslash D$ of the hypersurface

$$
\begin{array}{ll}
D:=\bigcup_{(i, j) \in B} D_{i, j} \subset \mathbb{T}^{r+1}, & D_{i, j}:=\left\{x_{i}=\alpha_{i, j}\right\}, \alpha_{i, j} \in \mathbb{C}^{*}, \\
& B:=\left\{(i, j) \mid 1 \leq j<n_{i}, 0 \leq i \leq r\right\} .
\end{array}
$$

Note that $X$ is homeomorphic to $\mathbb{C}_{n_{0}}^{*} \times \cdots \times \mathbb{C}_{n_{r}}^{*}$, the toric complex $\mathbb{T}_{K_{\mathbf{n}}}$ associated to the complete multipartite graph $K_{\mathbf{n}}$; the analytic structure of $X$ depend on the sets of $n_{i}-1$ points removed from each $\mathbb{C}^{*}$ factor. In fact, $f=f_{\mathbf{n}}^{\mathbf{d}}$ also depends on them, but they are omitted for notational simplicity.

Lemma 4.1. The map $f=f_{\mathbf{n}}^{\mathbf{d}}: X \rightarrow \mathbb{C}^{*}$ induces the group homomorphism $\chi:=\chi_{\mathbf{n}}^{\mathbf{d}}: N^{\chi} \rightarrow \mathbb{Z}$. 
Let $a \in \mathbb{C}^{*}$. Then the fiber $p^{-1}(a)=\left\{x_{0}^{d_{0}} \cdot \ldots \cdot x_{r}^{d_{r}}=a\right\}$ is a connected hypertorus ambient isomorphic to the hypertorus $\left\{y_{0}=a\right\}$, via a monomial automorphism $x_{j} \mapsto \prod_{j=0}^{r} y_{i}^{a_{i j}}$, determined by a unimodular integer matrix $A=$ $\left(a_{i j}\right)_{0 \leq i, j \leq r}$. Clearly the map $p$ is a trivial fiber bundle with fiber a connected torus. Let $C:=\left\{\mathbf{j}=\left(j_{0}, \ldots, j_{r}\right) \mid 1 \leq j_{i}<n_{i}, 0 \leq i \leq r\right\}$.

Proposition 4.2. The bifurcation set $\Lambda_{f}$ consists of the values

$$
\left\{a_{\mathbf{j}}:=\alpha_{0, j_{0}} \cdot \ldots \cdot \alpha_{r, j_{r}}\right\}_{\mathbf{j} \in C} .
$$

For generic points $\left\{\alpha_{i, j}\right\}$ the map $f$ has exactly $m:=\left(n_{0}-1\right) \cdot \ldots \cdot\left(n_{r}-1\right)$ distinct special fibers $f^{-1}\left(a_{\mathbf{j}}\right)$.

Proof. The hypersurface $D$ determines a stratification of the ambient torus $\mathrm{T}^{r+1}$ as follows. For each $I \subset B$ consider the intersection $D_{I}=\bigcap_{(i, j) \in I} D_{i, j}$; the strata are defined as differences of these closed sets. Then the top stratum is $X$ itself and the positive codimension strata are the intersections $D_{I}$ away from lower dimensional $D_{I^{\prime}}$.

Now $f: X \rightarrow \mathbb{C}^{*}$ is a restriction of $p$ which is a trivial fiber bundle. In order to apply the Thom Isotopy Lemma, it is enough to compactify $X$ as a subspace of (the normalization of)

$$
Z:=\left\{\left(\left[x_{0}: \cdots: x_{r}: y\right], t\right) \in \mathbb{P}^{r+1} \times \mathbb{C}^{*} \mid x_{0}^{d_{0}} \cdot \ldots \cdot x_{r}^{d_{r}}=t y^{d_{r+1}}\right\},
$$

with

$$
d_{r+1}=\sum d_{i},
$$

and extend $p$ to a proper map $Z \rightarrow \mathbb{C}^{*}$. Note that $p$ defines a trivial fibration on the strata at infinity.

In order to make $f$ into a fiber bundle we only have to remove those values $a \in \mathbb{C}^{*}$ (and their preimages from $X$ ) such that $p^{-1}(a)$ does not intersect transversally at least one stratum of the above stratification of $\mathbb{T}^{r+1}$. It is immediate that the fibers $p^{-1}(a)$ intersect $D_{I}$ transversally away from lower dimensional $D_{I^{\prime}}$, unless $D_{I}$ are already zero-dimensional. In that case, the stratum is simply a point which may lie or not on $p^{-1}(a)$. More precisely, $D_{I}$ is a point if and only if $I=I_{\left(j_{0}, \ldots, j_{r}\right)}=\left\{\left(0, j_{0}\right), \ldots,\left(r, j_{r}\right)\right\}$ for $\left(j_{0}, \ldots, j_{r}\right) \in C$. Note that $I_{\left(j_{0}, \ldots, j_{r}\right)} \subset f^{-1}\left(\alpha_{0, j_{0}} \cdot \ldots \cdot \alpha_{r, j_{r}}\right)$ and hence, $\Lambda_{f}$ is determined.

Finally, if $\left\{\alpha_{i, j}\right\}_{(i, j) \in B}$ are generic, then the values $a=\alpha_{0, j_{0}} \cdot \ldots \cdot \alpha_{r, j_{r}}$ are pairwise distinct. 
For the sake of simplicity, we will first treat the situation where $d_{i}=1$ for all $i$. In that case we just write $f=f_{\mathbf{n}}$ and $p: \mathbb{T}^{r+1} \rightarrow \mathbb{C}^{*}, p(\mathbf{x})=x_{0} \cdot \ldots \cdot x_{r}$. The fibers $p^{-1}(a)=\left\{\mathbf{x} \in \mathbb{T}^{r+1} \mid x_{0} \cdot \ldots \cdot x_{r}=a\right\}$ of the product map are isomorphic to the $r$-torus $\mathbb{T}^{r}$ under the identifications

$$
\begin{aligned}
\left(x_{0}, \ldots, x_{r}\right) & \longmapsto\left(x_{1}, \ldots, x_{r}\right), \\
\left(x_{1}, \ldots, x_{r}\right) & \longmapsto\left(\frac{a}{x_{1} \cdot \ldots \cdot x_{r}}, x_{1}, \ldots, x_{r}\right) .
\end{aligned}
$$

Moreover one can identify $\mathbb{T}^{r}$ with $\mathbb{P}^{r} \backslash\left\{z_{0} \cdot \ldots \cdot z_{r}=0\right\}$, via

$$
\begin{aligned}
\left(x_{1}, \ldots, x_{r}\right) & \longmapsto\left[1: \prod_{j=1}^{r} x_{j}: \prod_{j=2}^{r} x_{j}: \cdots: x_{r-1} x_{r}: x_{r}\right], \\
{\left[z_{0}: \cdots: z_{r}\right] } & \longmapsto\left(\frac{z_{1}}{z_{2}}, \ldots, \frac{z_{r}}{z_{0}}\right) .
\end{aligned}
$$

Thus, composing the inverse of the identifications (4.3)-(4.4), we obtain a diffeomorphism from $\mathbb{P}^{r} \backslash\left\{z_{0} \cdot \ldots \cdot z_{r}=0\right\}$ to $p^{-1}(a)$ provided by

$$
\left[z_{0}: \cdots: z_{r}\right] \longmapsto\left(a \frac{z_{0}}{z_{1}}, \frac{z_{1}}{z_{2}}, \ldots, \frac{z_{r}}{z_{0}}\right) .
$$

Lemma 4.3. Consider the map $f=f_{\mathbf{n}}: X \rightarrow \mathbb{C}^{*}$ as defined above. The fiber $F=f^{-1}(a)$ over $a \in \mathbb{C}^{*}$ is homeomorphic to the complement in $\mathbb{P}^{r}$ of the hyperplane arrangement $\mathcal{A}_{a}$ defined by the polynomial

$$
z_{0} \cdot \ldots \cdot z_{r} \cdot \prod_{j=1}^{n_{0}-1}\left(a z_{0}-\alpha_{0, j} z_{1}\right) \cdot\left(\prod_{i=1}^{r-1} \prod_{j=1}^{n_{i}-1}\left(z_{i}-\alpha_{i, j} z_{i+1}\right)\right) \cdot \prod_{j=1}^{n_{r}-1}\left(z_{r}-\alpha_{r, j} z_{0}\right),
$$

Proof. Under the identification

$$
\left\{\mathbf{x} \in \mathbb{T}^{r+1} \mid x_{0} \cdot \ldots \cdot x_{r}=a\right\} \longrightarrow \mathbb{P}^{r} \backslash\left\{x_{0} \cdot \ldots \cdot x_{r}=0\right\}
$$

described in (4.5) the fiber

$$
F=f^{-1}(a)=\left\{\mathbf{x} \in \mathbb{T}^{r+1} \mid x_{0} \cdot \ldots \cdot x_{r}=a, x_{i} \neq \alpha_{i, j}\right\}
$$

is sent to

$$
\left\{\left[z_{0}: z_{1}: \cdots: z_{r}\right] \in \mathbb{P}^{r} \mid z_{i} \neq 0, a z_{0} \neq \alpha_{0, j} z_{1}, z_{i} \neq \alpha_{i, j} z_{i+1}, z_{r} \neq \alpha_{r, j} z_{0}\right\} .
$$

Remark 4.4. By Proposition 4.2, we have that $m=\prod_{i=0}^{r}\left(n_{i}-1\right) \geq m^{\prime}:=\# \Lambda_{f}$. Let us denote

$$
\mathbb{C}_{m^{\prime}+1}^{*}=\mathbb{C}^{*} \backslash \Lambda_{f}^{*}, \quad E=\prod_{i=0}^{r} \mathbb{C}_{n_{i}}^{*} \backslash f^{-1}\left(\Lambda_{f}^{*}\right)
$$


The restriction $f=f_{\mathbf{n}}: E \rightarrow \mathbb{C}_{m^{\prime}+1}^{*}$ is a locally trivial fibration. For the generic values of Proposition 4.2 we have $m=m^{\prime}$. For non-generic values of the $\alpha_{i, j}$ 's the cardinal of the bifurcation set could very well be smaller than $m$.

For example the map $f=f_{3, \ldots, 3}:\left(\mathbb{C}^{*} \backslash\{ \pm 1\}\right)^{r+1} \rightarrow \mathbb{C}^{*}$ will have $\Lambda_{f}=\{ \pm 1\}$, thus $m^{\prime}=2<2^{r+1}=m$. More generally, one may take the set of $\left\{\alpha_{i, j}\right\}_{1 \leq j<n_{i}}$ to consist of the subgroup $\mu_{n_{i}-1}$ of roots of unity of order $n_{i}-1$ in $\mathbb{C}^{*}$.

We assume from now that $m=m^{\prime}$. The exact sequence in homotopy of the fibration $f=f_{\mathbf{n}}: E \rightarrow \mathbb{C}_{m+1}^{*}$ gives a short exact sequence of groups

$$
1 \longrightarrow \pi_{1}(F) \longrightarrow \pi_{1}(E) \longrightarrow \pi_{1}\left(\mathbb{C}_{m+1}^{*}\right) \longrightarrow 1,
$$

where $F$ is the fiber of $f$. Identify $\pi_{1}\left(\mathbb{C}_{m+1}^{*}\right)$ with the free group of rank $m+1$ written as $\mathbb{Z} * \mathbb{F}_{m}$, where $\mathbb{Z}$ is generated by the class $t$ of a meridian around the origin, and $\mathbb{F}_{m}$ is the free group on $a_{\mathbf{j}}$ (coming from meridians around the points in $\left.\Lambda_{f}\right), \mathbf{j} \in C$.

Note that $\pi_{1}(E)$ surjects onto the Artin group $A=\prod_{i=0}^{r} \mathbb{F}_{n_{i}}$, and the epimorphism is induced by the inclusion $E \hookrightarrow \prod_{i=0}^{r} \mathbb{C}_{n_{i}-1}^{*}$. The inclusion $\mathbb{C}^{*} \backslash \Lambda_{f} \hookrightarrow \mathbb{C}^{*}$ induces an epimorphism $\mathbb{Z} * \mathbb{F}_{m} \rightarrow \mathbb{Z}$ that satisfies $t \mapsto 1$ and $a_{\mathbf{j}} \mapsto 0$. Comparing the short exact sequence of the fiber bundle with the one defining the BestvinaBrady group, we obtain a commutative diagram whose vertical arrows are epimorphisms. In fact we have the following.

Lemma 4.5. The fundamental group of the arrangement defined in (4.6) is isomorphic to the Bestvina-Brady group $N=N_{\mathbf{n}}$ for almost every $a \in \mathbb{C}^{*}$.

Remark 4.6. The particular case $f=f_{2, \ldots, 2}:\left(\mathbb{C}^{*} \backslash\{1\}\right)^{r+1} \rightarrow \mathbb{C}^{*}$ leads to interesting hyperplane arrangements. The fiber $F=f^{-1}(a)$ over $a \neq 1$ is homeomorphic to the complement of the hyperplane arrangement $\mathcal{A}_{a}$ in $\mathbb{P}^{r}$ defined by $z_{0} z_{1} \ldots z_{r}\left(a z_{0}-z_{1}\right)\left(z_{1}-z_{2}\right) \ldots\left(z_{r-1}-z_{r}\right)\left(z_{r}-z_{0}\right)$. The bifurcation set $\Lambda_{f}$ is just $\lambda=1$. If $r>2$ then it is readily seen that the fundamental group of the complement to $\mathcal{A}_{a}$ does not change even when $a=1$. Lemma 4.5 implies that $N_{2, \ldots, 2}$ is the group of the graphic arrangement defined by the polynomial

$$
z_{0} z_{1} \ldots z_{r}\left(z_{0}-z_{1}\right)\left(z_{1}-z_{2}\right) \ldots\left(z_{r-1}-z_{r}\right)\left(z_{r}-z_{0}\right), \quad r>2 .
$$

Proof of Lemma 4.5. We have the following commutative diagram, where the rows are exact and the vertical arrows are surjective:

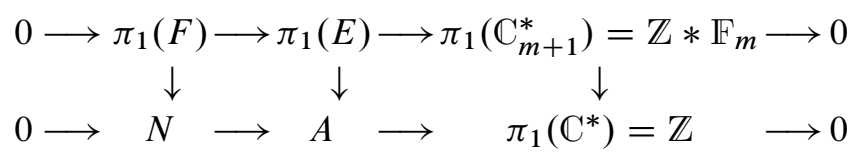


Let us fix $\mathbf{j}:=\left(j_{0}, \ldots, j_{r}\right) \in C$. Let $\gamma_{\mathbf{j}}$ be a lift of $a_{\mathbf{j}}$ to $\pi_{1}(E)$ that becomes trivial in the Artin group $A$. Let $G$ be the subgroup of $\pi_{1}(E)$ normally generated by $\gamma_{\mathbf{j}}$, $\mathbf{j} \in C$. If we identify $\pi_{1}(F)$ as a subgroup of $\pi_{1}(E)$, then

$$
K:=\operatorname{ker}\left(\pi_{1}(F) \longrightarrow N\right)=G \cap \pi_{1}(F) .
$$

It can be easily checked that $K$ is normally generated by $\left[g, \gamma_{\mathbf{j}}\right], g \in \pi_{1}(F), \mathbf{j} \in C$. We need to prove that $K$ is trivial, i.e., $g^{\gamma_{\mathbf{j}}}=g$ for all $g$ in $\pi_{1}(F)$ and $\mathbf{j} \in C$. Let us prove this claim.

The crucial observation is that the conjugation by $\gamma_{\mathbf{j}}$ is obtained by means of the monodromy action of $a_{\mathbf{j}}$ on $\pi_{1}(F)$; this is due to the choice of the lift $\gamma_{\mathbf{j}}$ to be trivial in $A$. In order to understand the action, we need to understand the behavior of $f$ near the bifurcation value $\alpha_{\mathbf{j}}$. The only stratum not transversal to $f^{-1}\left(\alpha_{\mathbf{j}}\right)$ is the stratum $I_{\mathbf{j}}$ which consists of a point $p_{\mathbf{j}}:=\left(\alpha_{0, j_{0}}, \ldots, \alpha_{r, j_{r}}\right)$. Let $\mathbb{D}_{\mathbf{j}}$ be a small closed disk around $\alpha_{\mathbf{j}}$ and let us denote $\mathbb{D}_{\mathbf{j}}^{*}:=\mathbb{D}_{\mathbf{j}} \backslash\left\{\alpha_{\mathbf{j}}\right\}$. The disk is chosen in order to ensure the following facts,

- The monomial map $p$ defined in (4.1) has a small Milnor ball $\mathbb{B}_{\mathbf{j}} \subset p^{-1}\left(\mathbb{D}_{\mathbf{j}}\right)$ for the point $p_{\mathbf{j}}$.

- $X_{\mathbf{j}}:=\overline{\phi^{-1}\left(\mathbb{D}_{\mathbf{j}}\right) \backslash \mathbb{B}_{\mathbf{j}}}$ fibers trivially over $\mathbb{D}_{\mathbf{j}}$.

We have realized a decomposition $f^{-1}\left(\mathbb{D}_{\mathbf{j}}^{*}\right)=X_{\mathbf{j}}^{*} \cup \check{\mathbb{B}}_{\mathbf{j}}$ for $X_{\mathbf{j}}^{*}:=X_{\mathbf{j}} \cap E$ and $\check{\mathbb{B}}_{\mathbf{j}}:=\mathbb{B}_{\mathbf{j}} \cap E$. The intersections $Y_{\mathbf{j}}:=X_{\mathbf{j}} \cap \check{\mathbb{B}}_{\mathbf{j}} Y_{\mathbf{j}}^{*}:=X_{\mathbf{j}}^{*} \cap \breve{\mathbb{B}}_{\mathbf{j}}$ are arcwise connected.

Since the statement deals with equalities of type $g^{\gamma_{\mathbf{j}}}=g$, we may choose as $F$ any non-special fiber. Let us fix $\tilde{\alpha}_{\mathbf{j}} \in \partial \mathbb{D}_{\mathbf{j}}$ and fix $F:=f^{-1}\left(\tilde{\alpha}_{\mathbf{j}}\right)$; for later use, let us denote $F_{\mathbf{j}}:=f^{-1}\left(\alpha_{\mathbf{j}}\right)$, which is a special fiber. Let us denote $M:=F \cap \check{\mathbb{B}}_{\mathbf{j}}$, $M_{\mathbf{j}}:=F \cap \breve{B}_{\mathbf{j}}, \breve{F}:=F \cap X_{\mathbf{j}}, \breve{F}_{\mathbf{j}}:=F_{\mathbf{j}} \cap X_{\mathbf{j}}, F^{\partial}=M \cap \breve{F}$ and $F_{\mathbf{j}}^{\partial}=M_{\mathbf{j}} \cap \breve{F}_{\mathbf{j}}$.

By the above construction,

$$
X_{\mathbf{j}} \cong \check{F} \times \mathbb{D}_{\mathbf{j}} \cong \breve{F}_{\mathbf{j}} \times \mathbb{D}_{\mathbf{j}}, \quad X_{\mathbf{j}}^{*} \cong \check{F} \times \mathbb{D}_{\mathbf{j}}^{*}
$$

and these homeomorphisms restrict to

$$
Y_{\mathbf{j}} \cong F^{\partial} \times \mathbb{D}_{\mathbf{j}} * \cong F_{\mathbf{j}}^{\partial} \times \mathbb{D}_{\mathbf{j}}, \quad Y_{\mathbf{j}}^{*} \cong F^{\partial} \times \mathbb{D}_{\mathbf{j}}^{*} ;
$$

moreover the pairs $\left(\check{F}, F^{\partial}\right)$ and $\left(\check{F}_{\mathbf{j}}, F_{\mathbf{j}}^{\partial}\right)$ are isotopic in $X_{\mathbf{j}}$. We can choose a representative of $\gamma_{\mathbf{j}}$ in $Y_{\mathbf{j}}^{*}$; the above homeomorphism imply that the action of $\gamma_{\mathbf{j}}$ by conjugation on $\pi_{1}(\breve{F})$ is trivial. Hence, in view of the Seifert-van Kampen theorem, it is enough to check that the action of $\gamma_{\mathbf{j}}$ by conjugation on $\pi_{1}(M)$ is also trivial. 
This is done as follows. Using the Taylor expansion of $p$ around $p_{\mathbf{j}}$ we can choose analytic coordinates $\left(y_{0}, \ldots, y_{r}\right)$ for $\mathbb{B}_{\mathbf{j}}$ (centered at $p_{\mathbf{j}}$ ) and for $\mathbb{D}_{\mathbf{j}}$ (centered at $\alpha_{\mathbf{j}}$ after a translation) such that $p\left(y_{0}, \ldots, y_{r}\right)=y_{0}+\cdots+y_{r}$.

Then, $M$ has the homotopy type of the complement of the arrangement defined by the equation $z_{1} \cdot \ldots \cdot z_{r} \cdot\left(z_{1}+\cdots+z_{r}-b\right)=0$ in $\mathbb{C}^{r}$, for some $b \in \mathbb{C}^{*}$. The group $\pi_{1}(M)$ is generated by the meridians of the hyperplanes and it is abelian (since $r \geq 2$ ). Let $\mu$ be the meridian of one of the hyperplanes; the element $\mu^{\gamma_{\mathbf{j}}}$ is constructed geometrically using the monodromy around $a_{\mathbf{j}}$ and it is again a meridian of the same hyperplane. Since the group is abelian we obtain that $\mu^{\gamma_{\mathbf{j}}}=\mu$; since the group $\pi_{1}(M)$ is generated by these meridians we obtain that $\gamma_{\mathbf{j}}$ acts trivially on $\pi_{1}(M)$ as required.

Proof of Theorem 3.2. It is an immediate consequence of Lemmas 4.3 and 4.5.

Proof of Corollary 3.3. In order to obtain the arrangement $\mathcal{A}_{\mathbf{n}}$ in $\mathrm{P}^{2}$ we only need to take a generic 2-dimensional slice of the hyperplane arrangement $\mathcal{A}_{a}$ in $\mathbb{P}^{r}$.

We now look at the general case of $f=f_{\mathbf{n}}^{\mathbf{d}}$, where $d_{i} \geq 1$ are integers whose greatest common divisor is 1 . The fibers of the product map $p=p^{\mathbf{d}}: \mathbb{T}^{r+1} \rightarrow \mathbb{C}^{*}$ are still tori isomorphic to $\mathbb{T}^{r}$, but the identification map does not have an explicit form in terms of the $d_{i}$ 's. In fact, the torus $\mathbb{T}^{r}$ naturally lives as $\mathbb{P}^{r}(\mathbf{d}) \backslash\left\{z_{0} \cdots z_{r}=\right.$ 0 ) inside the weighted projective space $\mathbb{P}^{r}(\mathbf{d})$. Therefore the fibers of $f$ can be identified with complements of hypersurfaces in $\mathbb{P}^{r}(\mathbf{d})$.

Remark 4.7. In case one of the $d_{i}$ 's is equal to 1 , say $d_{0}=1$, we can still realize the fibers of $f$ as complements of hypersurfaces in $\mathbb{P}^{r}$. Indeed, the fiber $p^{-1}(a)=$ $\left\{x_{0} \cdot x_{1}^{d_{1}} \cdot \ldots \cdot x_{r}^{d_{r}}=a\right\} \subset \mathbb{T}^{r+1}$ is homeomorphic to $\mathbb{P}^{r} \backslash\left\{z_{0} \cdot \ldots \cdot z_{r}=0\right\}$ via the mapping: $x_{i}=z_{i} z_{i+1}^{-1}$ for $1 \leq i \leq r$, and $x_{0}=a z_{0}^{d_{r}} z_{1}^{-d_{1}} z_{2}^{d_{1}-d_{2}} \cdots z_{r}^{d_{r-1}-d_{r}}$.

Example 4.8. Consider the map $f=f_{2, \ldots, 2}^{\mathbf{d}}:\left(\mathbb{C}^{*} \backslash\{1\}\right)^{r+1} \rightarrow \mathbb{C}^{*}$ and assume $d_{0}=1$. The bifurcation set is $\Lambda_{f}=\{1\}$. The fiber $F=f^{-1}(a)$ is homeomorphic to the complement of the arrangement of hypersurfaces $\mathcal{A}_{a}$ in $\mathbb{P}^{r}$ defined by the polynomial

$$
z_{0} \cdots z_{r} \cdot \prod_{i=1}^{r}\left(z_{i}-z_{i+1}\right) \cdot \prod_{j=1}^{n_{0}-1}\left(a z_{0}^{d_{r}}-z_{1}^{d_{1}} z_{2}^{d_{2}-d_{1}} \cdots z_{r}^{d_{r}-d_{r-1}}\right) .
$$


Proof of Theorem 3.4. The proof follows the same ideas as the proof of Theorem 3.2. We have seen above that the generic fibers of $f$ are in a natural way hypersurfaces of some weighted projective space. The special fibers are obtained as in Proposition 4.2; they correspond to some 0-dimensional non-zero strata. If we take out the special fibers we obtain a short exact sequence like (4.7). The local behavior around the bad 0-dimensional strata is like in the proof of Lemma 4.5 and the result follows.

\section{Fibrations and homotopy groups}

We will show here that the map

$$
f: X=\mathbb{T}^{r+1} \backslash D \longrightarrow \mathbb{C}^{*},
$$

as the restriction of the monomial map

$$
p: \mathbb{T}^{r+1} \longrightarrow \mathbb{C}^{*}
$$

given in (4.1), is in fact like a fibration in codimension 1. Also, in order to investigate the homology of the Artin kernels $N_{\Gamma}^{\chi}$, we construct geometric approximations of their $K(\pi, 1)$ complexes.

Recall that

$$
f: f^{-1}(B)=X_{\Lambda} \longrightarrow \mathbb{C}^{*} \backslash \Lambda=B
$$

is a fiber bundle. The bifurcation set $\Lambda$ consists of the elements $a \in \mathbb{C}^{*}$ for which the fiber $p^{-1}(a)$ is not transverse to all the strata of the divisor $D$. Also recall from the proof of Proposition 4.2 that these non-transversal strata are zero-dimensional and, since the stratification is finite, their union is a finite set.

5.1. Quasifibrations. In order to describe the objects and concepts mentioned above we need some definitions. Let $f: X \rightarrow C$ be a surjective map where $C$ is path-connected. For $c \in C$, let us denote

$$
F_{c}:=f^{-1}(c) .
$$

Definition 5.1 ([15]). A map $f: X \rightarrow C$ is a quasifibration (resp. an $n$-quasifibration) if it induces a weak homotopy equivalence (resp. $n$-equivalence)

$$
f:\left(X, F_{c}\right) \longrightarrow(C, c), \quad \text { for all } c \in C,
$$


that is, if for any $x \in F_{c}$ the morphism

$$
f_{*}: \pi_{i}\left(X, F_{c}, x\right) \longrightarrow \pi_{i}(C, c)
$$

is an isomorphism for all $i \geq 0$ (resp. for all $i<n$ and a surjection for $i=n$ ).

A characterization of $n$-quasifibrations is the following (cf. [22, 17]): a map $f: X \rightarrow C$ is an $n$-quasifibration if and only if the inclusion $F_{c} \rightarrow H_{c}$ of any fiber into the homotopy theoretic fiber $H_{c}$ is a (weak) homotopy $n$-equivalence.

As a consequence of this, all the fibers of an $n$-quasifibration have the same weak homotopy type up to dimension $n$.

5.2. Monomial quasifibrations. We will show that the monomial maps $f: X=$ $\mathbb{T}^{r+1} \backslash D \rightarrow \mathbb{C}^{*}$ considered in the previous section, $D$ as in (4.2), have a quasifibration structure. For the rest of the section we assume $r \geq 3$ unless otherwise stated.

Proposition 5.2. The map

$$
f: X=\mathbb{T}^{r+1} \backslash D \longrightarrow \mathbb{C}^{*}
$$

defined by

$$
f(\mathbf{x})=x_{0}^{d_{0}} \cdot \ldots \cdot x_{r}^{d_{r}}
$$

is an r-quasifibration.

Proof. The proof will use the tools for quasifibrations devised in the proof of the Dold-Thom theorem as outlined in Hatcher [18, Chapter 4, Section 4.K]. The restriction $f: X_{\Lambda} \rightarrow \mathbb{C}^{*} \backslash \Lambda=B$ is a fiber bundle, and thus a fibration and a quasifibration. Choose neighborhoods $B_{j}$ of the points $q_{j}$ in the finite set $\Lambda$ so that $B \cup \bigcup_{j} B_{j}$ is a cover of $\mathbb{C}^{*}$. The result will follow from the gluing theorem in [18, Lemma 4K.3] (see also [17, Theorem 2.3]) as soon as we prove that the restrictions $f: f^{-1}\left(B_{j}\right) \rightarrow B_{j}$ are $r$-quasifibrations.

Recall that to each $q_{j} \in \Lambda$ it corresponds a non-transversality point $p_{j}$ in the intersection of $D$ with the fiber $f^{-1}(\lambda)$ of $f: \mathbb{T}^{r+1} \rightarrow \mathbb{C}^{*}$. Using [18, Corollary $4 \mathrm{~K} .2]$, it is enough to prove the following local statement. For any $q_{j} \in \Lambda$, there exist neighborhoods $U_{j}$ of $p_{j}$ in $\mathbb{T}^{r+1}$ and $V_{j}$ of $q_{j}$ in $\mathbb{C}^{*}$ such that $f: X_{j}=$ $U_{j} \cap X \rightarrow V_{j}$ is an $r$-quasifibration.

As in Lemma 4.5, in local coordinates centered at $p_{j}$, respectively $q_{j}$, we have to consider the map $f: \mathbb{T}^{r+1} \rightarrow \mathbb{C}$ given by $f(z)=d_{0} z_{0}+\cdots+d_{r} z_{r}$. The fibers of this map $F_{c}=f^{-1}(c)$ are complements to hyperplane arrangements:

$$
F_{c}=\mathbb{C}^{r} \backslash\left\{z_{1} \ldots z_{r}\left(d_{1} z_{1}+\cdots+d_{r} z_{r}-c\right)=0\right\} .
$$


The arrangements involved are in general position as in the work of Hattori [19]. The results there give that $F_{c}$ has the following homotopy type

$$
F_{c} \simeq \begin{cases}\left(T^{r}\right)^{(r)} & \text { if } c \neq 0, \\ \left(T^{r-1}\right)^{(r-1)} & \text { if } c=0,\end{cases}
$$

where $T^{k}$ is the real $k$-dimensional torus, and $\left(T^{k}\right)^{(l)}$ is its $l$-skeleton. That yields $\pi_{1}\left(F_{c}\right)=\mathbb{Z}^{r}$ and

$$
\pi_{i}\left(F_{c}\right)=0 \text { if } \begin{cases}1<i<r & \text { and } c \neq 0, \\ 1<i<r-1 & \text { and } c=0 .\end{cases}
$$

The long exact homotopy sequence of the pair $\left(\mathbb{T}^{r+1}, F_{c}\right)$ provides the vanishing and surjection needed to obtain that the map $f:\left(\mathbb{T}^{r+1}, F_{c}\right) \rightarrow(\mathbb{C}, c)$ is an $r$-equivalence. Hence $f: \mathbb{T}^{r+1} \rightarrow \mathbb{C}$ is an $r$-quasifibration, and the local statement is proved.

Corollary 5.3. Let $F$ be any fiber of the map $f: X=\mathbb{T}^{r+1} \backslash D \rightarrow \mathbb{C}^{*}$. Then $\pi_{i}(F)=0$ for $1<i<r-1$.

Proof. By the previous Proposition, $f$ is an $r$-quasifibration, with aspherical base and aspherical total space. The claim follows from the long exact homotopy sequence of $f$.

Proposition 5.4. Let $F$ be the general fiber of the map $f: X=\mathbb{T}^{r+1} \backslash D \rightarrow \mathbb{C}^{*}$, $r \geq 2$. Then $\pi_{r-1}(F)=0$.

Proof. Note that $f: E \rightarrow B$ is a fiber bundle with fiber $F$ and aspherical base $B=\mathbb{C}^{*} \backslash \Lambda$. Thus we are done if we show that $\pi_{r-1}(E)=0$.

Suppose that the map $f: X=\mathbb{T}^{r+1} \backslash D \rightarrow \mathbb{C}^{*}$ defined by $f(\mathbf{x})=x_{0}^{d_{0}} \cdot \ldots \cdot x_{r}^{d_{r}}$ has $m$ special fibers $\Lambda=\left\{a_{1}, \ldots, a_{m}\right\}$ (see Proposition 4.2). In this case, $E=$ $X \backslash \bigcup_{j=1}^{m}\left\{x_{0}^{d_{0}} \cdot \ldots \cdot x_{r}^{d_{r}}=a_{j}\right\}$.

Consider now the divisor $\bar{D}=D \cup \bigcup_{j=1}^{m}\left\{x_{r+1}-a a_{j}^{-1}=0\right\}$ in $\mathbb{T}^{r+2}$, for some $a \in \mathbb{C}^{*}$. It is readily seen that $E$ can be identified with the special fiber $\bar{F}_{a}=\bar{f}^{-1}(a)$ of the map $\bar{f}: \bar{X}=\mathbb{T}^{r+2} \backslash \bar{D} \rightarrow \mathbb{C}^{*}$ given by $\bar{f}\left(\mathbf{x}, x_{r+1}\right)=$ $x_{0}^{d_{0}} \cdot \ldots \cdot x_{r}^{d_{r}} x_{r+1}$. On the other hand, $\pi_{r-1}\left(\bar{F}_{a}\right)=0$ by Corollary 5.3 , and so we are done. 
Let $F$ be the general fiber of the map $f: X=\mathbb{T}^{r+1} \backslash D \rightarrow \mathbb{C}^{*}$ defined by $f(\mathbf{x})=x_{0}^{d_{0}} \cdot \ldots \cdot x_{r}^{d_{r}}$, where $D=\bigcup_{i=0}^{r} \bigcup_{j=1}^{n_{i}-1}\left\{x_{i}=\alpha_{i, j}\right\}$. Then $X$ is clearly homotopy equivalent to $T_{\Gamma} \subset T^{r+1}$ the toric complex associated with the multipartite graph $\Gamma=K_{\mathbf{n}}$. Let $T_{\Gamma}^{\chi}$ be the infinite cyclic cover of $T_{\Gamma}$ associated with the epimorphism $\chi: A_{\Gamma} \rightarrow \mathbb{Z}$ defined by $\chi\left(\sigma_{i, j}\right)=d_{i}$. The multiplication map $f: T_{\Gamma} \rightarrow \mathbb{S}^{1}$, now seen in the real context, is a fibration whose fiber has the homotopy type of $T_{\Gamma}^{\chi}$. Recall that $T_{\Gamma}^{\chi}$ is an Eilenberg-MacLane space for the Artin kernel $N_{\Gamma}^{\chi}=\operatorname{ker} \chi$.

Corollary 5.5. For $r \geq 2$, the general fiber $F$ is a smooth quasi-projective variety of dimension $r$ that has the homotopy type of an $r$-dimensional $C W$-complex. The $r$-skeleton of the infinite cyclic cover $T_{\Gamma}^{\chi}$ has the homotopy type of $F$. In particular

$$
H_{i}\left(N_{\Gamma}^{\chi}\right) \cong \begin{cases}H_{i}(F) & \text { for } i \leq r, \\ \pi_{r}^{*}(F), & \text { for } i=r+1,\end{cases}
$$

where $\pi_{i}^{*}$ are the coinvariants of $\pi_{i}$ under the $\pi_{1}$-action.

Proof. We know that $\pi_{1}(F)=N_{\Gamma}^{\chi}$. From Corollary 5.3 and Proposition 5.4 we have that $\pi_{i}(F)=0$ for $1<i<r$. That ensures that $\left(T_{\Gamma}^{\chi}\right)^{(r)}$ and $F$ are homotopy equivalent.

We obtain that the first non-vanishing homotopy group of the general fiber $\pi_{r}(F)$ is of infinite rank, as $\pi_{r}^{*}(F)$ is so.

All the special fibers $F_{s}$ are smooth $r$-dimensional quasi-projective varieties sharing the homotopy type of an $r$-dimensional CW-complex. If $r \geq 3$, the $(r-1)$ skeletons of $F_{s}$ and of the general fiber $F$ have the same homotopy type.

Corollary 5.6. The special fiber $F_{s}$ is a smooth $r$-dimensional quasi-projective variety. If $r=2$, the special fiber $F_{s}$ is aspherical and $\pi_{1}\left(F_{s}\right)$ is not isomorphic to $N_{\Gamma}^{\chi}$. If $r \geq 3$, we have that

$$
\pi_{i}\left(F_{S}\right) \begin{cases}=N_{\Gamma}^{\chi}, & \text { if } i=1, \\ =0, & \text { if } 1<i<r-1, \quad H_{i}\left(N_{\Gamma}^{\chi}\right) \cong H_{i}\left(F_{S}\right) \text { for } i<r \\ \neq 0 & \text { if } i=r-1,\end{cases}
$$

and an exact sequence

$$
H_{r}\left(F_{s}\right) \longrightarrow H_{r}\left(N_{\Gamma}^{\chi}\right) \longrightarrow \pi_{r-1}^{*}\left(F_{s}\right) \longrightarrow 0 .
$$

In other words, if $r \geq 3$, then $\pi_{r-1}^{*}\left(F_{S}\right)$ is a finitely generated abelian group of rank $b_{r}\left(F_{s}\right)-b_{r}(F)$. 
Proof. We know that $\pi_{1}\left(F_{S}\right)=N_{\Gamma}^{\chi}$, if $r \geq 3$. From Corollary 5.3 we have that $\pi_{i}\left(F_{S}\right)=0$ for $1<i<r$. That ensures $F_{s}^{(r-1)}=F^{(r-1)}=\left(T_{\Gamma}^{\chi}\right)^{(r-1)}$ up to homotopy. The exact sequence (5.1) comes from the long exact sequence in homology obtained from the classifying map $F_{s} \rightarrow T_{\Gamma}^{\chi}=K\left(N_{\Gamma}^{\chi}, 1\right)$.

\section{References}

[1] E. Artal, J. I. Cogolludo-Agustín, and D. Matei, Quasi-projectivity, Artin-Tits groups, and pencil maps. In J. I. Cogolludo-Agustín and E. Hironaka (eds.), Topology of algebraic varieties and singularities. Contemporary Mathematics, 538. American Mathematical Society, Providence, R.I., and Real Sociedad Matemática Española, Madrid, 2011. 113-136. Zbl 1221.14022 Zbl 1210.14004 (collection) MR 2777818 MR 2777813 (collection)

[2] M. Bestvina, Questions in geometric group theory. Preprint 2000 (update 2004). http://www.math.utah.edu/ bestvina/eprints/questions-updated.pdf

[3] M. Bestvina and N. Brady, Morse theory and finiteness properties of groups. Invent. Math. 129 (1997), no. 3, 445-470. Zbl 0888.20021 MR 1465330

[4] R. Bieri, Normal subgroups in duality groups and in groups of cohomological dimension 2. J. Pure Appl. Algebra 7 (1976), no. 1, 35-51. Zbl 0322.20024 MR 0390078

[5] R. Bieri, Homological dimension of discrete groups. $2^{\text {nd }}$ ed., Queen Mary College Mathematical Notes. Queen Mary College, Department of Pure Mathematics, London, 1981. Zbl 0357.20027 (1 ${ }^{\text {st }}$ ed.) MR 0715779

[6] K.-U. Bux and C. González, The Bestvina-Brady construction revisited: geometric computation of $\Sigma$-invariants for right-angled Artin groups. J. London Math. Soc. (2) 60 (1999), no. 3, 793-801. Zbl 025.20026 MR 1753814

[7] R. Charney and M. W. Davis, Finite $K(\pi, 1)$ s for Artin groups. In F Quinn (ed.), Prospects in topology. Annals of Mathematics Studies, 138. Princeton University Press, Princeton, N.J., 1995, 110-124. Zbl 0930.55006 Zbl 0833.00037 (collection) MR 1368655 MR 1368648 (collection)

[8] D. C. Cohen, M. Falk, and R. Randell, Discriminantal bundles, arrangement groups, and subdirect products of free groups. Preprint 2010. arXiv:1008.0417 [math.GT]

[9] C. De Concini and C. Procesi, On the geometry of toric arrangements. Transform. Groups 10 (2005), no. 3-4, 387-422. Zbl 1099.14043 MR 2183118

[10] C. De Concini and C. Procesi, Topics in hyperplane arrangements, polytopes and box-splines. Universitext, Springer, New York, 2011. Zbl 1217.14001 MR 2722776

[11] G. Denham and A. I. Suciu, Moment-angle complexes, monomial ideals and Massey products. Pure Appl. Math. Q. 3 (2007), no. 1. Special issue in honor of Robert D. MacPherson. Part 3, 25-60. Zbl 1169.13013 MR 2330154

[12] W. Dicks and I. J. Leary, Presentations for subgroups of Artin groups. Proc. Amer. Math. Soc. 127 (1999), no. 2, 343-348. Zbl 0923.20032 MR 1605948 
[13] A. Dimca, Ș. Papadima, and A. I. Suciu, Quasi-Kähler Bestvina-Brady groups. J. Algebraic Geom. 17 (2008), no. 1, 185-197. Zbl 1176.20037 MR 2357684

[14] A. Dimca, S. Papadima, and A. I. Suciu, Non-finiteness properties of fundamental groups of smooth projective varieties. J. Reine Angew. Math. 629 (2009), 89-105. Zbl 1170.14017 MR 2527414

[15] A. Dold and R. Thom, Quasifaserungen und unendliche symmetrische Produkte. Ann. of Math. (2) 67 (1958), 239-281. Zbl 0091.37102 MR 0097062

[16] P. A. Griffiths and J. Harris, Principles of algebraic geometry. Pure and Applied Mathematics. Wiley-Interscience, New York, 1978. Zbl 0408.14001 MR 0507725

[17] M. A. Guest, M. Kwieciński, and B. W. Ong, Pseudo vector bundles and quasifibrations. Hokkaido Math. J. 29 (2000), no. 1, 159-170. Zbl 0984.32011 MR 1745508

[18] A. Hatcher, Algebraic topology. Cambridge University Press, Cambridge, 2002. Zbl 1044.55001 MR 1867354

[19] A. Hattori, Topology of $C^{n}$ minus a finite number of affine hyperplanes in general position. J. Fac. Sci. Univ. Tokyo Sect. IA Math. 22 (1975), no. 2, 205-219. Zbl 0306.55011 MR 0379883

[20] M. Jambu and S.. Papadima, Deformations of hypersolvable arrangements. Topology Appl. 118 (2002), no. 1-2, 103-111. Zbl 0995.32017 MR 1877718

[21] D. Matei, Arrangements of hypersurfaces and Bestvina-Brady groups. Oberwolfach Rep. 4 (2007), no. 3, 2347-2348.

[22] J. P. May, Weak equivalences and quasifibrations. In R. A. Piccinini (ed.), Groups of self-equivalences and related topics. Lecture Notes in Mathematics, 1425. Springer, Berlin etc., 1990, 91-101. Zbl 0713.55010 Zbl 0695.00020 (collection) MR 1070579 MR 1070570 (collection)

[23] J. Meier, H. Meinert, and L. VanWyk, Higher generation subgroup sets and the $\Sigma$-invariants of graph groups. Comment. Math. Helv. 73 (1998), no. 1, 22-44. Zbl 0899.57001 MR 1610579

[24] L. Moci, A Tutte polynomial for toric arrangements. Trans. Amer. Math. Soc. 364 (2012), no. 2, 1067-1088. Zbl 1235.52038 MR 2846363

[25] M. Namba, Branched coverings and algebraic functions. Pitman Research Notes in Mathematics Series, 161, Longman Scientific \& Technical, Harlow, and John Wiley \& Sons, New York, N.Y., 1987. Zbl 0706.14017 MR 0933557

[26] P. Orlik and H. Terao, Arrangements of hyperplanes. Grundlehren der Mathematischen Wissenschaften, 300, Springer, Berlin etc., 1992. Zbl 0757.55001 MR 1217488

[27] S. Papadima and A. I. Suciu, Higher homotopy groups of complements of complex hyperplane arrangements. Adv. Math. 165 (2002), no. 1, 71-100. Zbl 1019.52016 MR 1880322

[28] S. Papadima and A. I. Suciu, When does the associated graded Lie algebra of an arrangement group decompose? Comment. Math. Helv. 81 (2006), no. 4, 859-875. Zbl 1104.52009 MR 2271225 
[29] S. Papadima and A. I. Suciu, Algebraic invariants for Bestvina-Brady groups. J. Lond. Math. Soc. (2) 76 (2007), no. 2, 273-292. Zbl 1104.52009 MR 2271225

[30] S. Papadima and A. I. Suciu, Toric complexes and Artin kernels. Adv. Math. 220 (2009), no. 2, 441-477. Zbl 1208.57002 MR 2466422

[31] R. Randell, Homotopy and group cohomology of arrangements. Topology Appl. 78 (1997), no. 3, 201-213. Zbl 0880.55007 MR 1454600

[32] J.-P. Serre, Sur la topologie des variétés algébriques en caractéristique $p$. Symposium internacional de topología algebraica. International symposium on algebraic topology, Universidad Nacional Autónoma de México and UNESCO, Mexico City, 1958, 24-53. Zbl 0098.13103 MR 0098097

[33] I. Shimada, Fundamental groups of algebraic fiber spaces. Comment. Math. Helv. 78 (2003), no. 2, 335-362. Zbl 1022.14008 MR 1988200

[34] J. Stallings, A finitely presented group whose 3-dimensional integral homology is not finitely generated. Amer. J. Math. 85 (1963), 541-543. Zbl 0122.27301 MR 0158917

[35] A. I. Suciu, Characteristic varieties and Betti numbers of free abelian covers. Int. Math. Res. Not. IMRN 2012 (2012), no. 4, 1063-1124. Zbl 06340344 MR 3168402

[36] C. T. C. Wall, Finiteness conditions for CW-complexes. Ann. of Math. (2) 81 (1965), 56-69. Zbl 0152.21902 MR 0171284

Received July 2, 2012

Enrique Artal Bartolo, Departamento de Matemáticas, IUMA,

Universidad de Zaragoza, c/ Pedro Cerbuna 12, E-50009 Zaragoza, Spain

e-mail: artal@unizar.es

José Ignacio Cogolludo-Agustín, Departamento de Matemáticas, IUMA, Universidad de Zaragoza, c/ Pedro Cerbuna 12, E-50009 Zaragoza, Spain e-mail: jicogo@unizar.es

Daniel Matei, Institute of Mathematics of the Romanian Academy, P.O. Box 1-764, RO-014700 Bucharest, Romania

e-mail: Daniel.Matei@imar.ro 